\title{
Konishi operator at intermediate coupling
}

\author{
Sergey Frolov*† \\ Hamilton Mathematics Institute and School of Mathematics, \\ Trinity College, Dublin 2, Ireland
}

\begin{abstract}
TBA equations for two-particle states from the $\mathfrak{s l}(2)$ sector proposed by Arutyunov, Suzuki and the author are solved numerically for the Konishi operator descendent up to 't Hooft's coupling $\lambda \approx 2046$. The data obtained is used to analyze the properties of $\mathrm{Y}$-functions and address the issue of the existence of the critical values of the coupling. In addition we find a new integral representation for the BES dressing phase which substantially reduces the computational time.
\end{abstract}

*Email: frolovs@maths.tcd.ie

${ }^{\dagger}$ Correspondent fellow at Steklov Mathematical Institute, Moscow. 


\section{Contents}

1. Introduction 1

2. Konishi state energy 4

3. Y-functions 9

4. Large $\lambda$ expansion from the numerical data 16

5. Conclusion 22

6. Appendix 23

\section{Introduction}

The Thermodynamic Bethe Ansatz (TBA) is an efficient tool to determine the finitesize spectrum of two-dimensional relativistic integrable models [1]. The relevance of the TBA for the $\mathrm{AdS}_{5} \times \mathrm{S}^{5}$ spectral problem was advocated in [2] where Lüscher's approach [3] was used to relate exponential corrections to string energy to wrapping effects in dual field theory. Its application to a nonrelativistic model requires understanding thermodynamic properties of a so-called mirror theory [4] which is obtained from the original model by means of a double-Wick rotation. The mirror model of the light-cone $\mathrm{AdS}_{5} \times \mathrm{S}^{5}$ superstring was studied in detail in [4] where, in particular, the asymptotic spectrum was identified, and the mirror form of the Bethe-Yang equations of [5] was determined.

The major step towards realizing the TBA approach is to formulate a so-called string hypothesis [6] which classifies the states contributing in the thermodynamic limit. TBA equations and the associated Y-system are then readily derived from it, see e.g. [7]. This step was made last year in [8] where the mirror Bethe-Yang equations [4] were used to formulate a string hypothesis for the $\mathrm{AdS}_{5} \times \mathrm{S}^{5}$ mirror model. This opened a way to derive the corresponding canonical $[9,10,11]$ and simplified TBA equations [12].

The TBA equations combined with a certain analytic continuation procedure proposed for relativistic models in $[13,14]$ can be also used to find energies of excited states. ${ }^{1}$ The procedure was called the contour deformation trick in [28] because

\footnotetext{
${ }^{1}$ For other approaches and applications, see e.g. [15]-[27].
} 
it basically reduces to deforming the integration contours in ground-state TBA equations while keeping their form untouched. As a result, excited states TBA equations differ only by integration contours. For practical applications however one should take the integration contours back to their ground-state positions, and this results in the appearance of state-dependent driving terms in the TBA equations.

The contour deformation trick was used in [28] to analyze two-particle states from the $\mathfrak{s l}(2)$ sector. It was shown there that two-particle states are divided into infinitely-many classes, each class having its own set of driving terms in the TBA equations. For the Konishi-like states the TBA equations of [28] are believed to be equivalent to those of $[11,29],{ }^{2}$ and our numerics strongly supports this.

The TBA equations obtained in [28] were used to derive the five-loop anomalous dimension of the Konishi operator, and to show numerically [33] that the corresponding result perfectly agrees with the one recently obtained via the generalized Lüscher formulae [34]. The analysis in [28, 33] was then extended to prove the agreement analytically first for the Konishi operator [35], and then for an arbitrary twist-two operator [36] reproducing the results in [37]. ${ }^{3}$ For other applications of the TBA approach to the AdS/CFT spectral problem, see [52]-[57].

In a parallel development the TBA equations proposed in [11] were used in [29] to compute anomalous dimension of the Konishi operator up to a relatively large value of the 't Hooft coupling constant $\lambda \approx 664$. Analyzing the results obtained, the authors of [29] found the following fitting function for the Konishi state energy or, equivalently, for the conformal dimension of the dual Konishi operator

$$
\bar{E}_{K}^{G K V}(\lambda)=\sqrt[4]{\lambda}\left(2.0004+\frac{1.988}{\sqrt{\lambda}}-\frac{2.60}{\lambda}+\frac{6.2}{\lambda^{3 / 2}}\right) .
$$

This formula allows one to make four remarkable predictions. First, it predicts that the coefficient $c_{-1}$ of the leading term in the large $\lambda$ expansion is equal to 2 which agrees with the spectrum of string theory in flat space [58] and asymptotic Bethe ansatz considerations [59]. Second, it shows the vanishing of the constant term $c_{0}$. Third, it makes a new prediction which disagrees with the semiclassical consideration in [60] that the first nonvanishing subleading coefficient is also equal to 2. And fourth,

\footnotetext{
${ }^{2}$ The set of integral equations proposed in [11, 29] was named an integral form of the Y-system. We find this terminology somewhat misleading because these integral equations were not derived from the Y-system conjectured in [30] but were proposed by following the pure TBA approach, that is by using the canonical TBA equations [9, 10,11], the contour deformation trick [13], and the large $J$ asymptotic solution of the Y-system [30]. A derivation of the TBA equations from the Ysystem requires understanding the complicated analytic structure of Y-functions, see $[9,31,28,32]$ for some results in this direction.

${ }^{3}$ The five-loop computations in [34, 37] were based on [38] where the four-loop anomalous dimension of the Konishi operator was computed and shown to agree with the direct field theory result $[39,40]$. This constituted a strong test of the AdS/CFT correspondence [41]. For other applications of Lüscher's approach, see e.g. [42]-[51].
} 
it predicts that up to an overall factor of $\sqrt[4]{\lambda}$ the Konishi state energy is a series in $1 / \sqrt{\lambda}$. The last prediction in fact agrees with the argumentation in [60] but it disagrees with the considerations in [61] where the free fermion model describing the $\mathfrak{s u}(1 \mid 1)$ sector in the semi-classical approximation was analyzed, and it was shown that the strong coupling expansion for short states is in powers of $1 / \sqrt[4]{\lambda}$. It is worth noting that this simple model indeed predicts that the constant term in the strong coupling expansion vanishes. The formulae derived in the framework of the free fermion model would definitely get quantum corrections, and if the prediction of [29] is correct it would imply that these corrections drastically change the structure of the strong coupling expansion.

In this paper we reconsider the computation of the Konishi state energy. We solve numerically the excited states TBA equations [28] for the Konishi operator up to 't Hooft's coupling $\lambda \approx 2046$, and use the data obtained to analyze the behavior of Y-functions. We use the analysis to address the issue of the existence of the critical values of $\lambda$ raised in [28]. The consideration in [28] was based on an assumption that the analytic properties of the exact Y-functions would follow those of the large $J$ asymptotic solution, and if this assumption is not realized then the TBA equations of $[29,28]$ formulated for Konishi-like states at weak coupling may be valid for all values of $\lambda$. Extrapolating our results we find that the first critical value for the Konishi operator is most probably greater than $5300, \lambda_{c r}^{(1)}>5300$, which is significantly higher than the estimate based on the large $J$ asymptotic solution that gives $\lambda_{c r}^{(1)} \approx 774$.

We also find that the contribution of $Y_{Q}$-functions to the Konishi state energy grows almost linearly with the string tension $g=\sqrt{\lambda} / 2 \pi$. If this behavior continues to hold for all values of $\lambda$ then this would imply that at large $\lambda$ the exact Bethe root asymptotes to a constant less than 2 . This would be drastically different from the asymptotic behavior of the corresponding solution of the Bethe-Yang equations and would, in particular, imply the absence of the critical values for the Konishi operator. This would be a very puzzling scenario because the full spectrum of string theory in flat space can be reproduced already from the Bethe-Yang equations [59], and if $w$ does not asymptote to 2 it would be necessary to explain how the spectrum follows from the TBA equations. In particular, the spectrum degeneracy would be more difficult to explain because Bethe roots of different states would not behave uniformly at strong coupling.

Examining the data obtained, we found convincing evidence in favor of the prediction of [29] that the first nonvanishing subleading coefficient $c_{1}$ of the $1 / \sqrt[4]{\lambda}$ term is equal to 2 , and the coefficient $c_{2}$ of the terms $1 / \sqrt{\lambda}$ vanishes. If one cuts the asymptotic series at order $1 / \lambda^{5 / 4}$ and fits our data then the coefficient $c_{1}$ appears to be $2.02 \pm 0.02$ depending on the fitting interval used. It is clearly reasonable to assume that $c_{1}$ is equal to 2 . Setting $c_{1}=0$, one then finds that $c_{2}=-0.02 \pm 0.01$ which is obviously very small.

Then, assuming from the very beginning that $c_{-1}=c_{1}=2$ and $c_{0}=c_{2}=0$ and 
fitting the data with $\lambda>77$, one gets the following fitting function for the Konishi state energy

$$
c_{-1}=c_{1}=2, c_{2}=0 \Longrightarrow \bar{E}_{K}(\lambda)=2 \sqrt[4]{\lambda}+\frac{2}{\sqrt[4]{\lambda}}-\frac{3.26}{\lambda^{3 / 4}}+\frac{2.53}{\lambda}+\frac{4.03}{\lambda^{5 / 4}},
$$

which differs from (1.1) by the presence of the $1 / \lambda$ term. Thus, the coefficient $c_{4}$ of the term $1 / \lambda$ does not vanish, and we cannot confirm the prediction of [29] that up to an overall factor of $\sqrt[4]{\lambda}$ the large $\lambda$ expansion of the Konishi state energy is a series in $1 / \sqrt{\lambda}$. The vanishing of $c_{2}$ could be related to the high degree of supersymmetry of the model as was pointed out in [60].

If on the other hand one follows [29] and assumes from the very beginning that $c_{2}=c_{4}=0$ then fitting the data with $\lambda>77$, one gets the following fitting function for the Konishi state energy

$$
c_{2}=c_{4}=0 \Longrightarrow \bar{E}_{K}(\lambda)=\sqrt[4]{\lambda}\left(2.00005+\frac{1.992}{\sqrt{\lambda}}-\frac{2.73}{\lambda}+\frac{7.45}{\lambda^{3 / 2}}\right)
$$

which obviously is in a very good agreement with (1.1). The coefficients in (1.3) mildly depend on the fitting interval used and, for example, the last coefficient can take values from 6 to 8 .

Let us also mention that our numerical results agree with those of [29] with the 0.0015 precision for most values of $\lambda$, and this implies the equivalence of the TBA equations of [11] and [28] for Konishi-like states at weak coupling. ${ }^{4}$

We used in our computation a new integral representation for the BES dressing phase [62] which significantly reduces the computational time.

The paper is organized as follows. In the next section we present the results of the numerical solution of the TBA equations for the Konishi state energy. In section 3 we discuss the properties of Y-functions and estimate the first critical value of $\lambda$. In section 4 we use our data to find the coefficients of the large $\lambda$ asymptotic expansion. In the appendix we describe the numerical algorithm used for the computation, and present new formulae for various dressing phases and kernels.

\section{Konishi state energy}

We solve numerically the following equations from [28]: the simplified TBA equations (4.2-4.5) for $Y_{M \mid w}, Y_{M \mid v w}$, and $Y_{ \pm}$-functions, and the hybrid equations (4.13) for $Y_{Q^{-}}$ functions, and determine the values of the Bethe root $u_{2}=-u_{1} \equiv w$ or, equivalently, the momentum $p=p(w)$ carried by a string particle from the exact Bethe equation

\footnotetext{
${ }^{4}$ Since the considerations in [28] and in v.3 of [11] have the same starting point - the mirror theory string hypothesis [8] - the equivalence of the TBA equations in fact follows from the equality of the mirror theory dressing phases used in [29] and [28] which was recently proven in [32].
} 
(8.61). The energy of the Konishi state is then given by the following formula

$$
E_{K}(\lambda)=2+2 \sqrt{1+4 g^{2} \sin ^{2} \frac{p}{2}}-\sum_{Q=1}^{\infty} \int \frac{d \widetilde{p}}{2 \pi} \log \left(1+Y_{Q}\right),
$$

where $\widetilde{p}$ is the momentum of a $Q$-particle of the mirror theory, and $g$ is the string tension related to 't Hooft's coupling $\lambda$ as $\lambda=4 \pi^{2} g^{2}$. The energy is given by the difference of the two terms - the first one is the contribution coming from the dispersion relation, and the second one is the $Y$-functions contribution. We denote the contributions as $E_{\text {dis }}$ and $E_{\mathrm{Y}}$, respectively, so that $E_{K}=E_{\mathrm{dis}}-E_{\mathrm{Y}}$. It is clear that $E_{\text {dis }}$ and $-E_{\mathrm{Y}}$ play the roles of the kinetic and potential energy of the Konishi state particles. We will also use the notation $E_{\mathrm{Y}_{\mathrm{Q}}}$ for the contribution of a $Y_{Q^{-}}$-function to the energy.

It is known that at large values of 't Hooft's coupling the energy of the Konishi state can be expanded in an asymptotic series in powers of $1 / \sqrt[4]{\lambda}$

$$
E_{K}(\lambda)=2 \sqrt[4]{\lambda}+c_{0}+\frac{c_{1}}{\sqrt[4]{\lambda}}+\frac{c_{2}}{\sqrt{\lambda}}+\frac{c_{3}}{\lambda^{3 / 4}}+\frac{c_{4}}{\lambda}+\frac{c_{5}}{\lambda^{5 / 4}}+\cdots
$$

where the leading $2 \sqrt[4]{\lambda}$ behavior follows from the string spectrum in flat space [58], and can be reproduced from the asymptotic Bethe ansatz [59]. The coefficient $c_{0}$ is believed to be equal to 0 because that is what one gets from both the free fermion model describing the $\mathfrak{s u}(1 \mid 1)$ sector and the asymptotic Bethe ansatz [61] but to our knowledge an honest string theory derivation of $c_{0}$ is absent. The large $\lambda$ perturbative expansion of the light-cone string sigma model (see [63] for a review) allows one to have any of the coefficients $c_{i}$ nonvanishing. It was however argued in [60] that the coefficient $c_{2}$ should vanish due to the high degree of supersymmetry of the model. Moreover, it follows from the fitting function (1.1) for the Konishi state energy obtained by solving the canonical TBA equations [29] that in fact both the coefficients $c_{2}$ and $c_{4}$ vanish, and then it is tempting to speculate that all coefficients with even indices vanish: $c_{2 k}=0$.

One of our aims is to demonstrate that the excited state TBA equations of [28] indeed predict that the leading coefficient is 2 , and then to understand if one can fix the coefficients $c_{k}$, and in particular if the numerical data indeed predicts that $c_{0}=c_{2}=c_{4}=0$.

The results of our computation of the Konishi state energy or, equivalently, the conformal dimension of the Konishi operator as a function of $g$ are collected in the table (6.1) from the Appendix. In Figure 1 we plot the data together with the graph of the function $E_{\text {dis }}\left(w_{\text {asym }}\right)$ where $w_{\text {asym }}=w_{\text {asym }}(\lambda)$ is the corresponding solution of the Bethe-Yang equation, and the graph of $2 \sqrt[4]{\lambda}$ which is the large $\lambda$ asymptote of both the exact and asymptotic energies. One sees that the exact energy graph approaches $2 \sqrt[4]{\lambda}$ faster than $E_{\text {dis }}\left(w_{\text {asym }}\right)$. It is also useful to plot the graph of $E_{\text {dis }}(w)$ as a function 


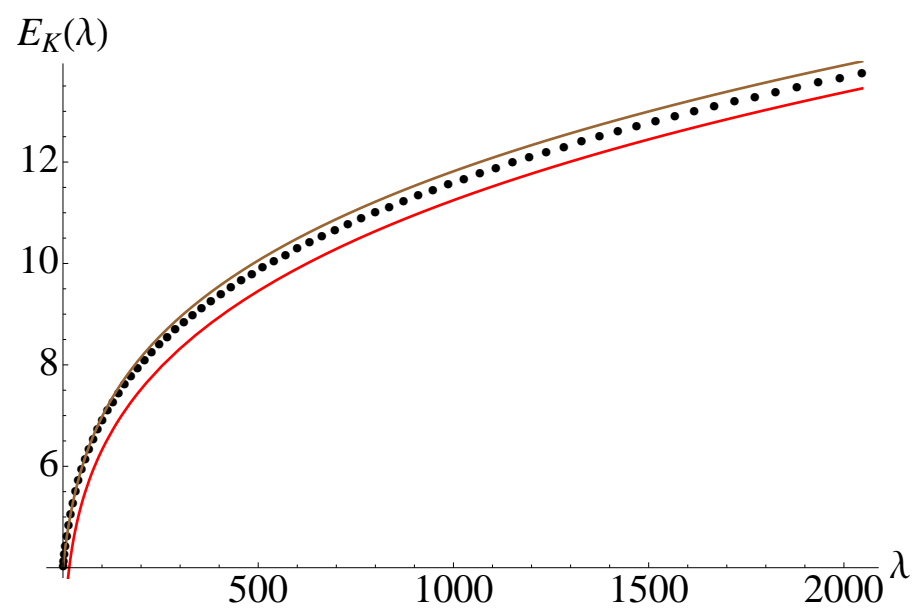

Figure 1: Black dots represent the numerical solution of the TBA equations for the Konishi state energy $E_{K}(\lambda)$. The brown (upper) curve represents the solution of the Bethe-Yang equation, and the red (lower) curve is the graph of $2 \sqrt[4]{\lambda}$ which is the large $\lambda$ asymptote of $E_{K}(\lambda)$. The range of the coupling constant is from $g=0.1, \lambda=0.39$ to $g=7.2, \lambda=2046.56$.

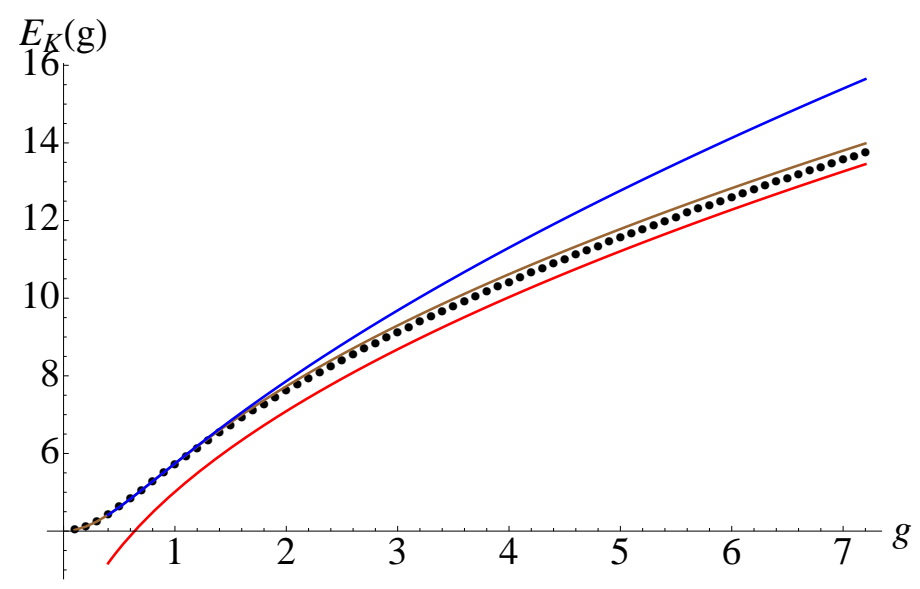

Figure 2: Here black dots, the brown and red curves are the same as in Figure 1, and the blue (upper) curve is the graph of $E_{\mathrm{dis}}(w)$ where $w=w(g)$ is the solution of the exact Bethe equations.

of $\lambda$ to compare its contribution with the exact energy. The corresponding plots are shown in Figure 2 where we use the string tension $g=\sqrt{\lambda} / 2 \pi$ as an independent variable. We see that $E_{\text {dis }}(w)$ grows almost linearly with $g$ for $g>4$ while the total energy grows as $\sqrt{g}$. This implies immediately that the Y-functions contribution also grows linearly, see Figure 3, and, moreover, the linear parts of $E_{\text {dis }}(w)$ and $E_{\mathrm{Y}}$ cancel each other leading to the $\sqrt{g}$ behavior of $E_{K}$. On the other hand, $E_{\text {dis }}\left(w_{\text {asym }}\right)$ grows only as $\sqrt{g}$ for these values of $g$. It is interesting that the linear dependence of $E_{\mathrm{Y}}$ becomes clearly visible already at very small values of $g$. To understand the reason for such a different behavior of $E_{\text {dis }}(w)$ and $E_{\text {dis }}\left(w_{\text {asym }}\right)$ we plot in Figure 4 the solutions of the exact Bethe equation and the Bethe-Yang equation for $w$ and 


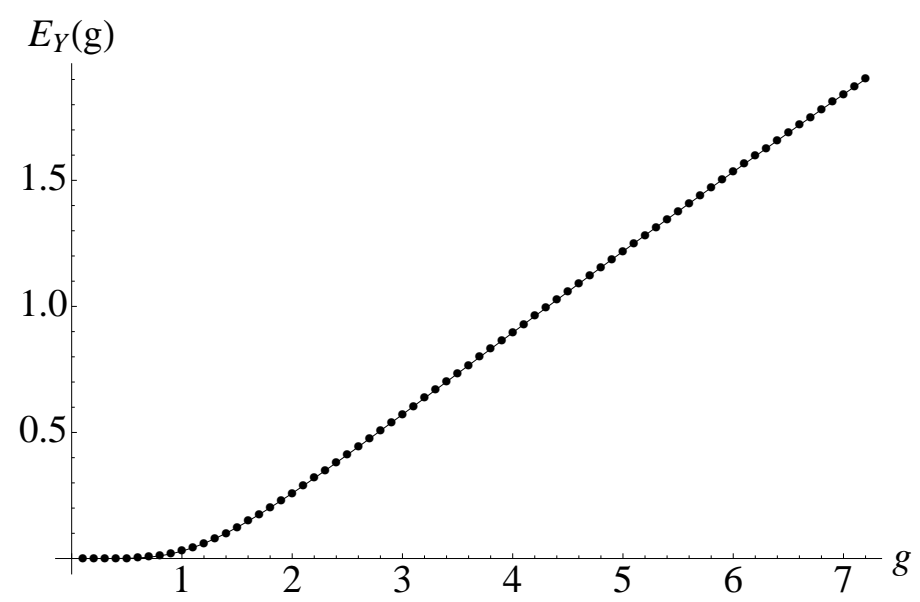

Figure 3: This is the graph of the contribution of $E_{\mathrm{Y}}$ to the energy. It obviously shows a linear growth starting already with $g \sim 2$.

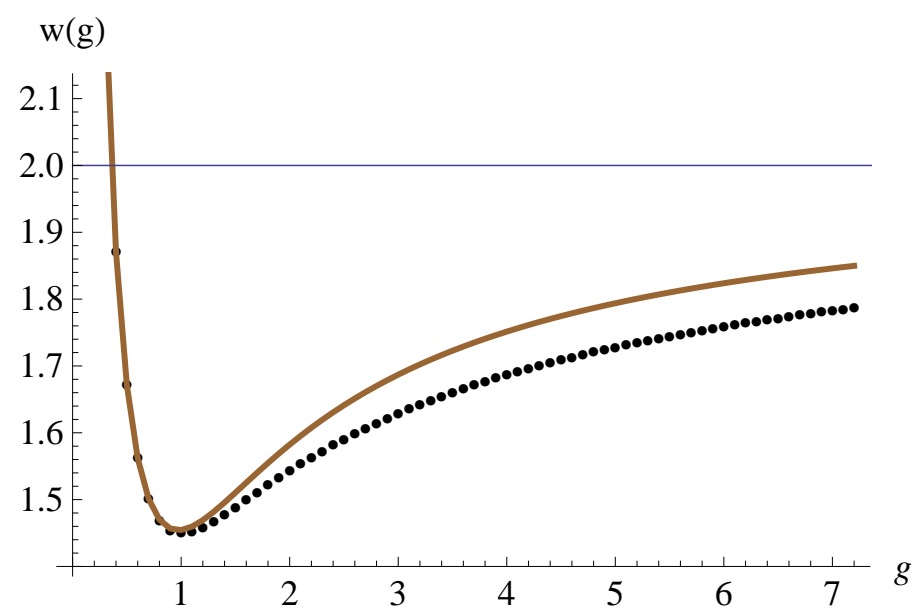

Figure 4: The black dots represent our numerical solution of the exact Bethe equation, and the brown curve is the graph of the corresponding solution of the Bethe-Yang equation. The exact Bethe root $w(g)$ reaches its minimum at $g \approx 1$, and $g \approx 1.6$ is the inflection point.

$w_{\text {asym }}$, respectively. The numerical data of the computation of the Bethe root $w$ are in the table (6.2) in Appendix.

One sees that $w$ is still pretty far from 2 which is the large $g$ asymptotic value of $w_{\text {asym }}$, and that the exact Bethe root is noticably smaller than $w_{\text {asym }}$. The corresponding exact momentum $p$ is not small at these values of $g$ and decreases much slower than $p_{\text {asym }}$. This explains why the dispersion relation contribution indeed grows as $g$.

It is tempting to conclude from Figures 2 and 3 that $E_{\text {dis }}(w)$ and $E_{\mathrm{Y}}$ would grow linearly for all values of $g$, and, therefore, $w$ in fact would asymptote to a constant $w_{\infty}$ less than 2. If this happens then this would make the existence of critical values of the coupling constant discussed in [28] rather improbable, and as a result the TBA 

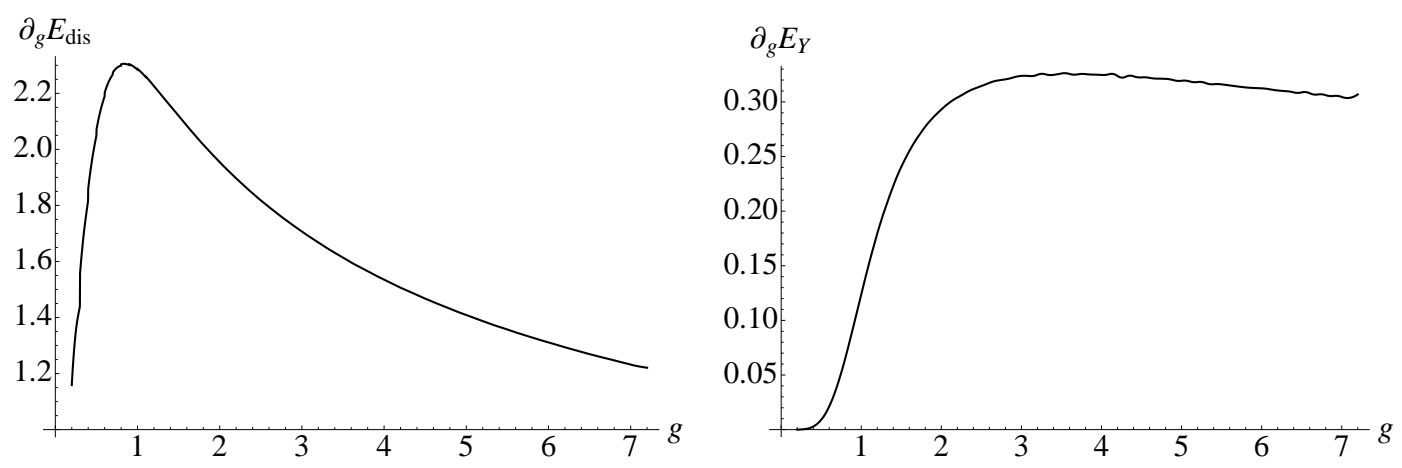

Figure 5: The graphs of the derivative of $E_{\text {dis }}$ and $E_{\mathrm{Y}}$ with respect to $g$.

equations for Konishi-like states [29, 28] might be valid for any value of $g$. Also, this would mean that the strong coupling limit of multi-particle states with finite number of particles and $J$ (short operators in dual field theory) is very different from the near flat space limit discussed in [59, 64] where the rapidities asymptote to 2, and one can study states with $J \sim \sqrt{g}$. The puzzle then is that the full spectrum of string theory in flat space can be reproduced already in the near flat space limit [59], and if $w$ does not asymptote to 2 it would be necessary to explain how the flat space string spectrum follows from the TBA equations. ${ }^{5}$

This is an intriguing scenario, and it would be very interesting to understand analytically if it is the one. Our numerics however seems to indicate that the linear growth of $E_{Y}$ might be a feature of the intermediate coupling regime we are studying, and it will slow down for larger values of $g$. In Figure 5 we plot the graphs of the derivative of $E_{\text {dis }}(w)$ and $E_{\mathrm{Y}}$ with respect to $g$ (obtained by using the Interpolation function in Mathematica). We see that the rate of change of $E_{\mathrm{Y}}$ reaches its maximum at $g \sim 3$, remains almost constant till $g \sim 4$ and then begins to decrease very slowly. We are not sure if this effect is genuine. The precision of our computation falls down for $g>4$, and the decrease in the rate of change of $E_{Y}$ may be just a numerical artifact. Since, as will be discussed in the next section, the contribution of an individual $Y_{Q}$-function to $E_{\mathrm{Y}}$ slightly decreases at large $g$ it might be also necessary to include the contribution of more $Y_{Q}$-functions than we did. Then, it is certainly possible that the rate of change of $E_{\mathrm{Y}}$ would stabilize at even larger values of $g$, and the scenario discussed above would be realized. The assumption that the Y-functions contribution would be linear in $g$ for very large $g$ has a profound consequence on the strong coupling dependence of the $Y_{1}$-function. Since $Y_{Q}$-functions are very small for $|u|>2$ the integration region in (2.1) is effectively of order $\sqrt{g}$ for large $g$, and, therefore, the Y-functions contribution would grow as $g$ only if $\log Y_{1}$ would be of order $\sqrt{g}$. However, in the next section we will see that it is not the

\footnotetext{
${ }^{5}$ Strictly speaking, even if $w$ asymptotes to 2 but with a rate different from the one of $w_{\text {asym }}$ it would be a challenge to derive the flat space string spectrum from TBA.
} 

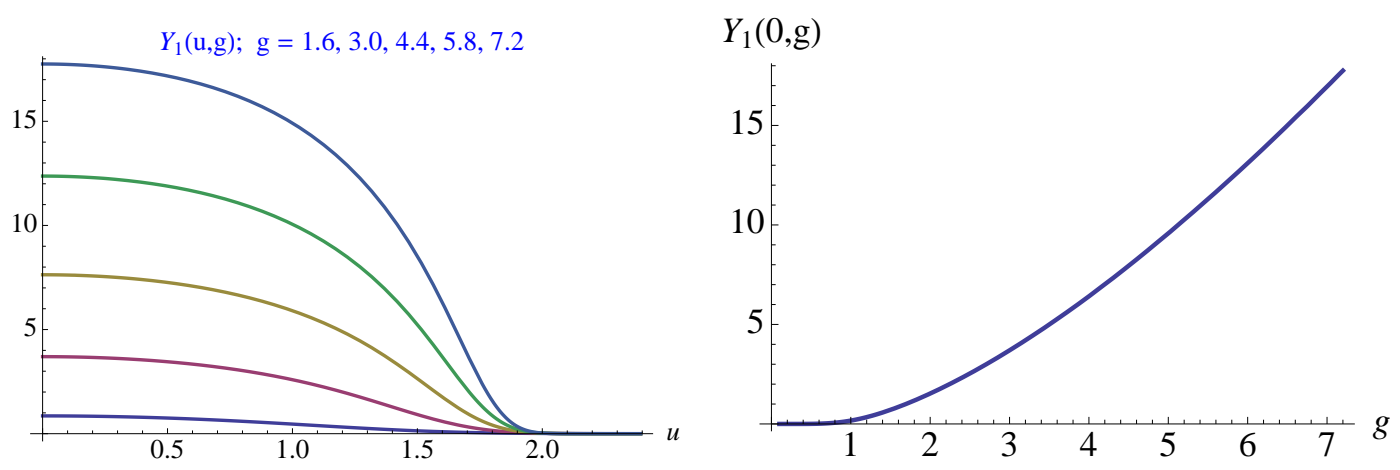

Figure 6: On the left figure graphs of five $Y_{1}$-functions with different values of $g$ are shown, and on the right figure the graph of $Y_{1}(0, g)$ as a function of $g$ is plotted.

case and for $g \sim 7, Y_{1}$ increases only as $g^{3 / 2}$. This behavior is different from both the asymptotic $Y_{1}$-function $g$-dependence and the exponential growth required by the scenario discussed above. This also shows clearly that the values of $g$ we have reached are not large enough, and we are still analyzing the intermediate coupling regime. What happens at larger values of $g$ remains to be understood.

\section{Y-functions}

In this section we discuss various properties of Y-functions. We begin with $Y_{Q^{-}}$ functions because the energy of the Konishi state depends explicitly on them.

In Figure 6 we show plots ${ }^{6}$ of several exact $Y_{1}$-functions computed at various values of $g$. One sees that $Y_{1}$ is getting larger in the interval $[-2,2]$ with $g$ increasing. In fact it increases very fast, and becomes much larger (one order of magnitude) than the asymptotic $Y_{1}^{o}$-function computed at the same values of $g$ and $w$. In particular, $Y_{1}(0, g)$ keeps increasing while the asymptotic $Y_{1}^{o}$-function computed with the exact Bethe root $w$ decreases at $u=0$ for $g>4$. To find the $g$-dependence we plot $\sqrt{g} Y_{1}(0, g)$ and $\sqrt{g} Y_{1}(1, g)$ in Figure 7 . We see that they are almost linear functions, and, therefore, $Y_{1} \sim g^{3 / 2}$. It is hardly possible that $Y_{1}$ would show the $g^{3 / 2}$-dependence for large $g$ because this would lead to the contribution of the order $\sqrt{g} \log g$ to the Konishi state energy which cannot be canceled by any reasonable contribution from $E_{\text {dis }}$. If the exact Bethe root $w$ approaches 2 for large $g$ then $Y_{1}(u, g)$ should asymptote to a finite function. The existence of the critical values of $g$ seems to require in addition that $Y_{1}(u, g)$ would go to 0 for any $u<2$ (but it could stay finite for $u \sim 2-\nu / g$ ). If $w$ approaches $w_{\infty}<2$ then, as was mentioned in the previous section, $\log Y_{1}$ must grow as $\sqrt{g}$.

In Figure 8 the plot of the contribution $E_{\mathrm{Y}_{1}}$ of $Y_{1}$-function to the Konishi state energy and the plot of its derivative $\partial_{g} E_{\mathrm{Y}_{1}}$ are shown. $E_{\mathrm{Y}_{1}}$ decreases too fast to be

\footnotetext{
${ }^{6}$ All Y-functions are even so we plot them only for $u \geq 0$.
} 

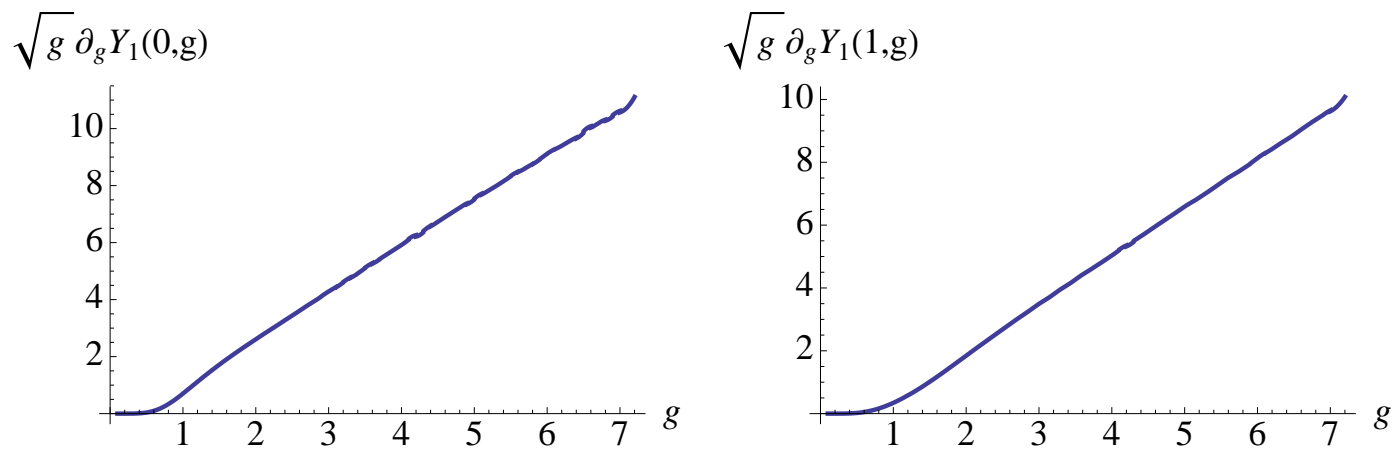

Figure 7: The graphs of $\sqrt{g} \partial_{g} Y_{1}(0, g)$ and $\sqrt{g} \partial_{g} Y_{1}(1, g)$ definitely show that $Y_{1}$ grows as $g^{3 / 2}$.
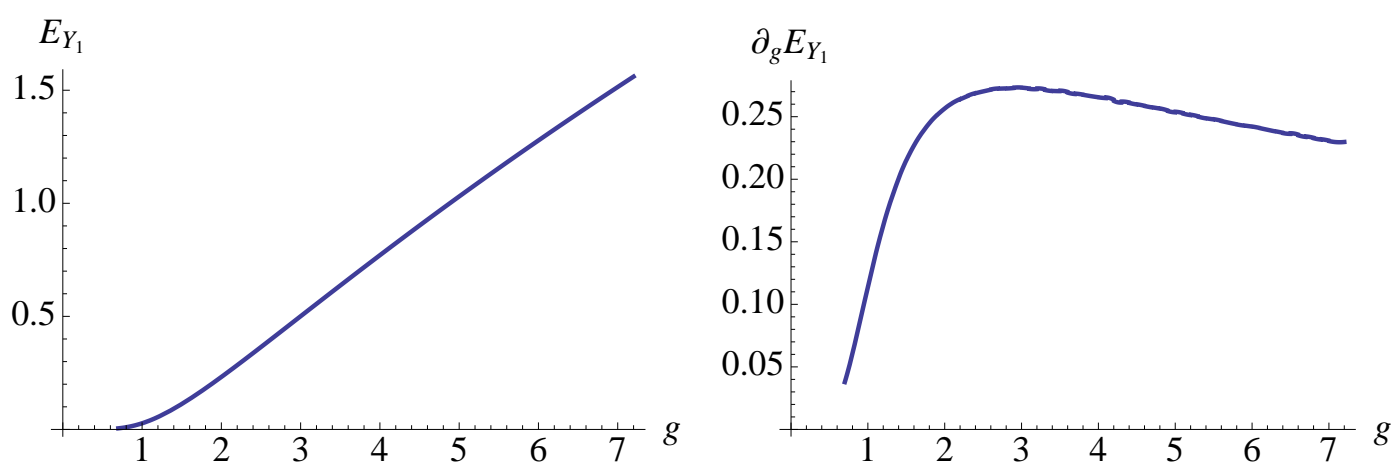

Figure 8: These are the plots of $E_{\mathrm{Y}_{1}}$ and $\partial_{g} E_{\mathrm{Y}_{1}}$.

explained by insufficient numerical precision. The scenario with $w_{\infty}<2$ would be realized only if $\partial_{g} E_{\mathrm{Y}_{1}}$ asymptotes to a positive constant.

Figure 9 shows plots of $Y_{2}$-functions. One sees that they exhibit a rather intricate $g$ dependence. The maximum value of $Y_{2}$ increases with $g$ and shifts to the right towards $u=2$.
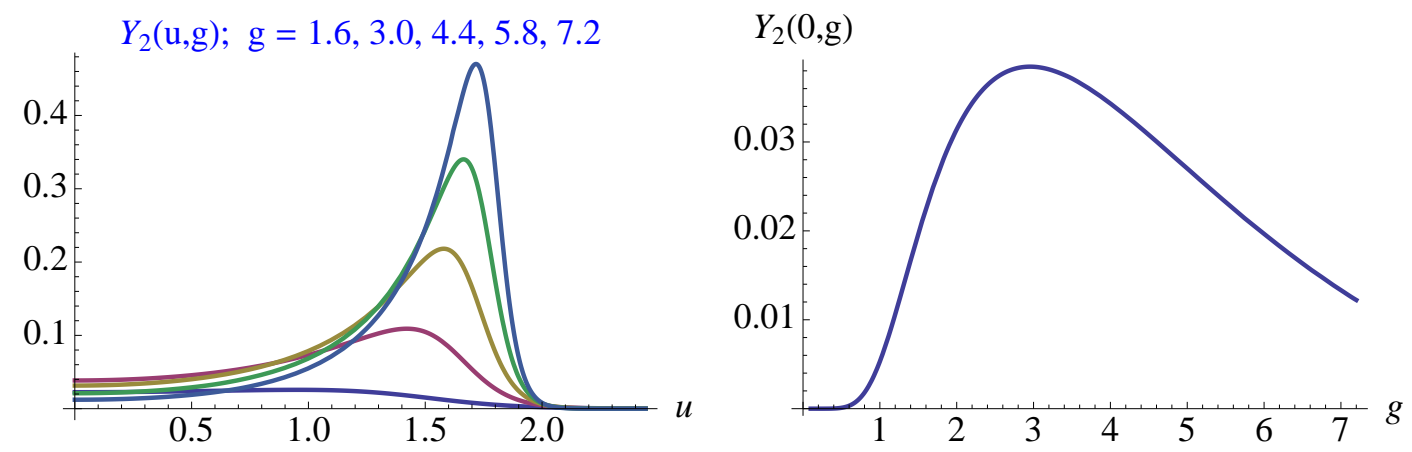

Figure 9: On the left figure graphs of five $Y_{2}$-functions with different values of $g$ are shown, and on the right figure the graph of $Y_{2}(0, g)$ as a function of $g$ is plotted. 
We see from Figure 9 that $Y_{2}(0, g)$ is decreasing for $g>3$. This behavior agrees with our expectations based on the analysis of asymptotic Y-functions. Let us recall that $Y_{2}$-function is one of the Y-functions that can be used to find the first subcritical value of $g$ because it vanishes at $u=0$ if $g=\bar{g}_{c r}^{(1)}$ [28]. Let us now assume that the critical value does exist. Then since $Y_{2}$ is non-negative for all values of $g$ it should have an expansion of the form

$$
Y_{2}(0, g) \sim\left(g-\bar{g}_{c r}^{(1)}\right)^{2}+\cdots
$$

Since most of our points are far from $\bar{g}_{c r}^{(1)}$, to estimate the first sub-critical value we first however use the linear fit. This would give the lowest estimate ${ }^{7}$ of the value of $\bar{g}_{c r}^{(1)}$ because $Y_{2}(0, g)$ has a double zero at $g=\bar{g}_{c r}^{(1)}$. Fitting our data in the interval $\left[g_{0}, 7.2\right]$ to the function $c_{1}\left(g-\bar{g}_{c r}^{(1)}\right)$, we get the results shown in (3.1).

\begin{tabular}{|c|c||c|c||c|c|}
\hline$g_{0}$ & Fit & $g_{0}$ & Fit & $g_{0}$ & Fit \\
\hline 6.2 & $-0.0061(g-9.19)$ & 6.3 & $-0.0060(g-9.22)$ & 6.4 & $-0.0059(g-9.24)$ \\
6.5 & $-0.0059(g-9.27)$ & 6.6 & $-0.0058(g-9.30)$ & 6.7 & $-0.0057(g-9.32)$ \\
6.8 & $-0.0057(g-9.35)$ & 6.9 & $-0.0056(g-9.38)$ & 7.0 & $-0.0055(g-9.40)$ \\
\hline
\end{tabular}

As expected, the estimated value of $\bar{g}_{c r}^{(1)}$ increases with $g_{0}$ approaching 7.2 , and one concludes from the table that $\bar{g}_{c r}^{(1)}>9$.4. If on the other hand one fits the data to $c_{2}\left(g-\bar{g}_{c r}^{(1)}\right)^{2}$, one gets

\begin{tabular}{|c|c||c|c||c|c|}
\hline$g_{0}$ & Fit & $g_{0}$ & Fit & $g_{0}$ & Fit \\
\hline 6.2 & $0.00061(g-11.66)^{2}$ & 6.3 & $0.00061(g-11.67)^{2}$ & 6.4 & $0.00061(g-11.67)^{2}$ \\
6.5 & $0.00061(g-11.68)^{2}$ & 6.6 & $0.00061(g-11.68)^{2}$ & 6.7 & $0.00060(g-11.69)^{2}$ \\
6.8 & $0.00060(g-11.70)^{2}$ & 6.9 & $0.00060(g-11.70)^{2}$ & 7. & $0.00060(g-11.71)^{2}$ \\
\hline
\end{tabular}

This fitting is much more stable then the linear one, and gives $\bar{g}_{c r}^{(1)} \sim 11.7$. It is certainly possible that decreasing $Y_{2}(0, g)$ would slow down for larger values of $g$ resulting in a larger estimate of $\bar{g}_{c r}^{(1)}$.

The plots of $E_{\mathrm{Y}_{2}}$ and $\partial_{g} E_{\mathrm{Y}_{2}}$ are shown in Figure 10. The rate of change of $E_{\mathrm{Y}_{2}}$ is still increasing, and one cannot make any reliable prediction about its strong coupling behavior.

Figure 10 shows similar plots of $Y_{3}$-function. It is still increasing at $u=0$ but it is clear that it will reach its maximum soon.

\footnotetext{
${ }^{7}$ We assume there will be no sharp changes in the behavior of $Y_{2}(0, g)$ for larger values of $g$.
} 

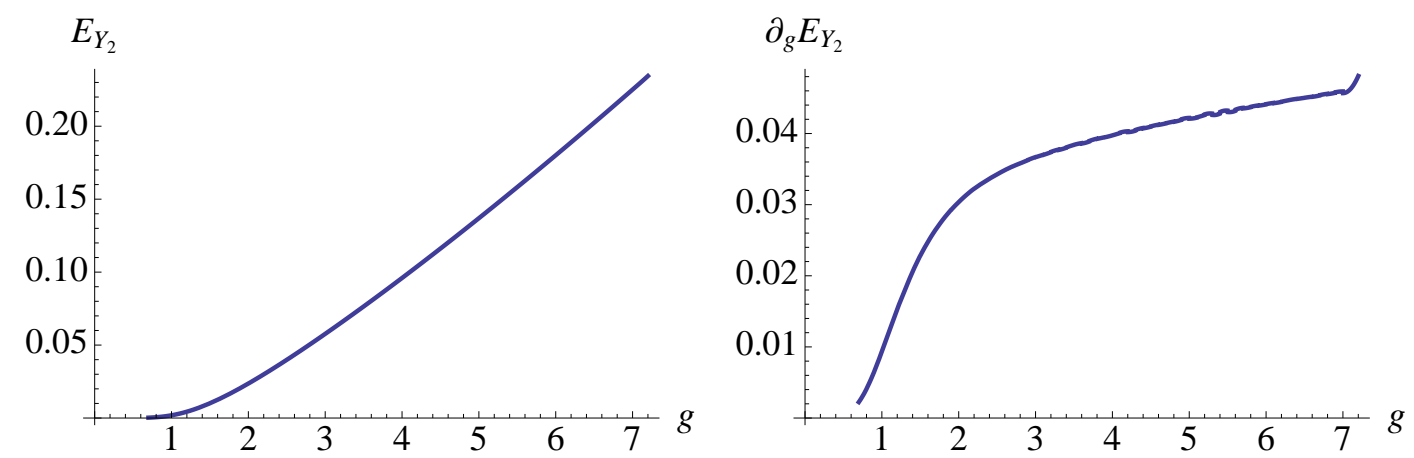

Figure 10: These are the plots of $E_{\mathrm{Y}_{2}}$ and $\partial_{g} E_{\mathrm{Y}_{2}}$.
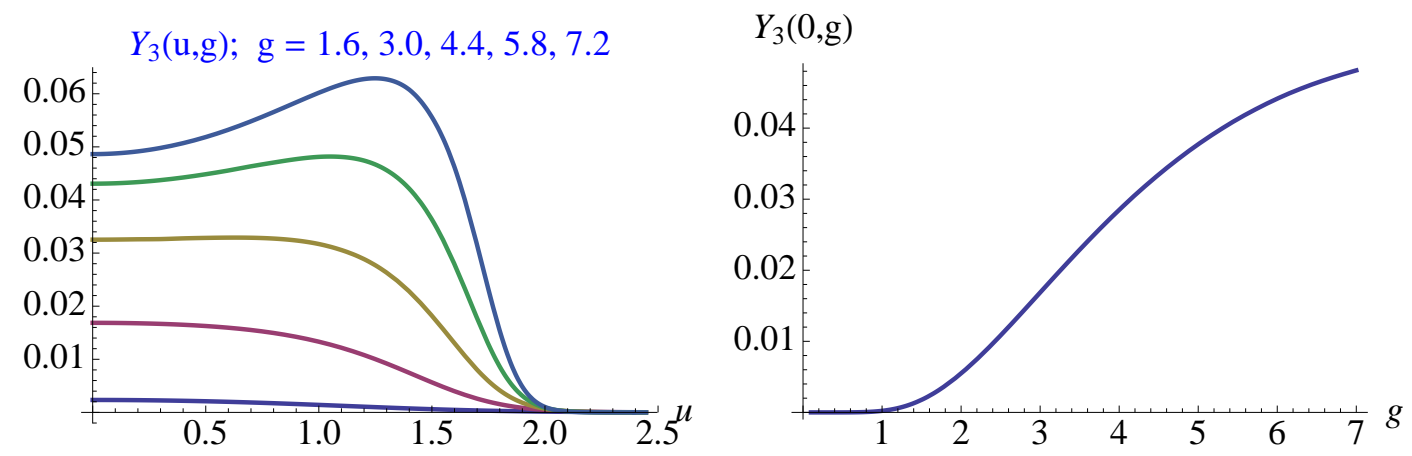

Figure 11: On the left figure graphs of five $Y_{3}$-functions with different values of $g$ are shown, and on the right figure the graph of $Y_{3}(0, g)$ as a function of $g$ is plotted.

Even though $Y_{3}$ is so small its contribution to the energy is also increasing linearly with $g$, see Figure 12, and moreover the rate of $E_{\mathrm{Y}_{3}}$ has already reached its maximum.
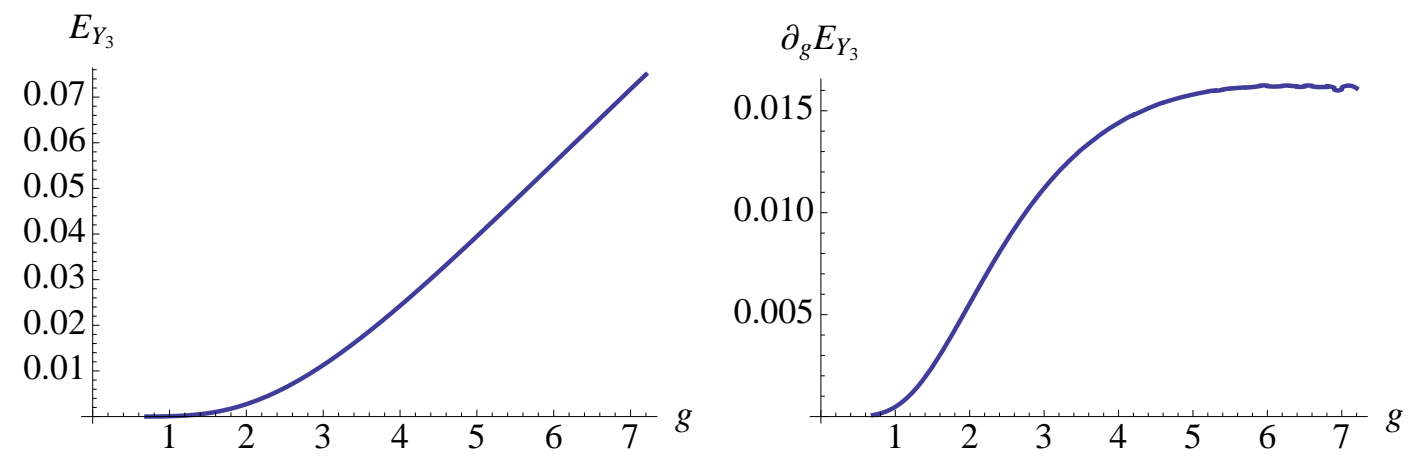

Figure 12: These are the plots of $E_{\mathrm{Y}_{3}}$ and $\partial_{g} E_{\mathrm{Y}_{3}}$.

Next we discuss $Y_{ \pm}$-functions, see Figures 13 and 14. The first subcritical value can be also determined from $Y_{ \pm}$because their values vanish at $u=0$, and they are 


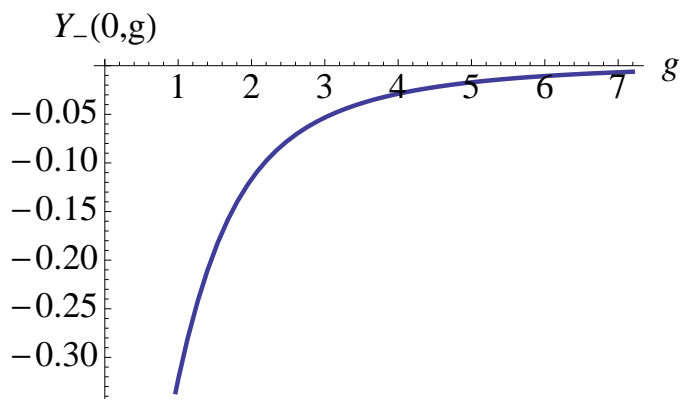

Figure 13: $Y_{-}$-functions

assumed to have the following expansions ${ }^{8}$

$$
Y_{ \pm}(0, g) \sim g-\bar{g}_{c r}^{(1)}+\cdots .
$$

Using again the linear fit, we get the following results for $Y_{-}(0, g)$

\begin{tabular}{|c|c||c|c||c|c|}
\hline$g_{0}$ & Fit & $g_{0}$ & Fit & $g_{0}$ & Fit \\
\hline 6.2 & $0.0035(g-8.93)$ & 6.3 & $0.0034(g-8.98)$ & 6.4 & $0.0033(g-9.03)$ \\
6.5 & $0.0033(g-9.08)$ & 6.6 & $0.0032(g-9.13)$ & 6.7 & $0.0031(g-9.18)$ \\
6.8 & $0.0030(g-9.23)$ & 6.9 & $0.0030(g-9.28)$ & 7. & $0.0029(g-9.33)$ \\
\hline
\end{tabular}

The results in the table (3.3) are obviously compatible with those in table (3.1) but the estimated subcritical values $\bar{g}_{c r}^{(1)}$ in (3.3) appear to be slightly less than the ones from (3.1). A better estimate of $\bar{g}_{c r}^{(1)}$ is obtained by fitting the data to $c_{1}\left(g-\bar{g}_{c r}^{(1)}\right)+c_{3}\left(g-\bar{g}_{c r}^{(1)}\right)^{3}$

\begin{tabular}{|c|c||c|c|}
\hline$g_{0}$ & Fit & $g_{0}$ & Fit \\
\hline 6.2 & $0.000045(g-11.14)^{3}+0.00087(g-11.14)$ & 6.3 & $0.000043(g-11.19)^{3}+0.00085(g-11.19)$ \\
6.4 & $0.000042(g-11.25)^{3}+0.00083(g-11.25)$ & 6.5 & $0.000041(g-11.30)^{3}+0.00081(g-11.30)$ \\
6.6 & $0.000040(g-11.36)^{3}+0.00079(g-11.36)$ & 6.7 & $0.000039(g-11.41)^{3}+0.00078(g-11.41)$ \\
6.8 & $0.000038(g-11.46)^{3}+0.00076(g-11.46)$ & 6.9 & $0.000037(g-11.51)^{3}+0.00074(g-11.51)$ \\
\hline
\end{tabular}

with the results similar to those from (3.2).

Since $Y_{+}(0, g)$ is still pretty far from 0 for the values of $g$ we are dealing with, its linear extrapolation to larger values of $g$ would not give very reliable results. Indeed, fitting our data for $Y_{+}(0, g)$ in the interval $\left[g_{0}, 7.2\right]$ to the linear function $c_{1}\left(g-\bar{g}_{c r}^{(1)}\right)$, we get the results shown in (3.5).

\begin{tabular}{|c|c||c|c||c|c|}
\hline$g_{0}$ & Fit & $g_{0}$ & Fit & $g_{0}$ & Fit \\
\hline 6.2 & $0.25(g-9.83)$ & 6.3 & $0.25(g-9.88)$ & 6.4 & $0.24(g-9.92)$ \\
6.5 & $0.24(g-9.97)$ & 6.6 & $0.24(g-10.01)$ & 6.7 & $0.23(g-10.05)$ \\
6.8 & $0.23(g-10.10)$ & 6.9 & $0.23(g-10.14)$ & 7. & $0.22(g-10.18)$ \\
\hline
\end{tabular}

Let us also mention that if one uses $c_{3}\left(g-\bar{g}_{c r}^{(1)}\right)^{3}$ as a fitting function one gets the largest of all the estimates: $\bar{g}_{c r}^{(1)}>16$.

Next we discuss $Y_{1 \mid v w}$-function, see Figure 15. This is the function that deter-

\footnotetext{
${ }^{8}$ It seems possible that the expansion could be of the form $Y_{ \pm}(0, g) \sim\left(g-\bar{g}_{c r}^{(1)}\right)^{3}+\cdots$.
} 

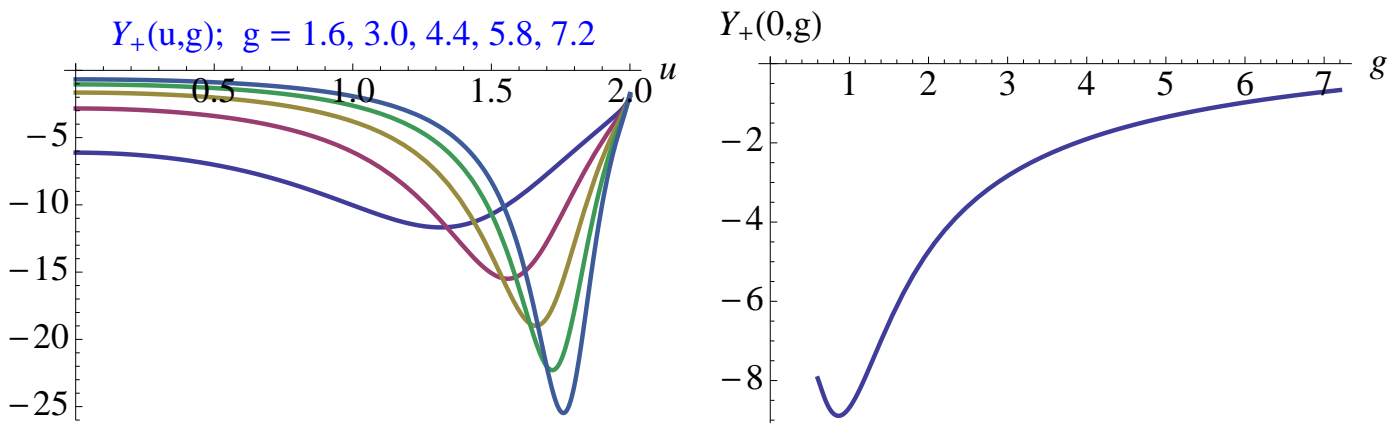

Figure 14: $Y_{+}$and $Y_{-}$-functions are approaching 0 at $u=0$ with $g$ increasing. For $g>\bar{g}_{c r}^{(1)}$ they become positive at $u=0$, and asymptote to their ground state value $Y_{ \pm}^{\text {grst }}=1$ for very large $g$.
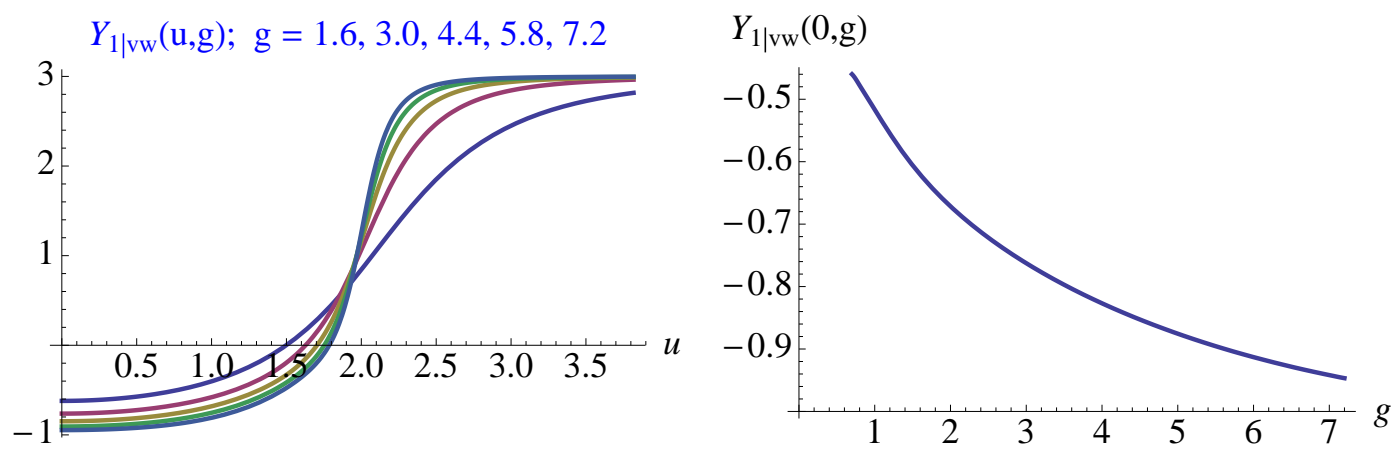

Figure 15: $Y_{1 \mid v w}$-function is approaching -1 at $u=0$ with $g$ increasing. It also approaches its ground state value at $u=\infty$ faster for larger $g$.

mines the first critical value of $g$ because its value at $u=0$ has the following behavior in the vicinity of $g=g_{c r}^{(1)}$

$$
Y_{1 \mid v w}(0, g)+1 \sim\left(g-g_{c r}^{(1)}\right)^{2}+\cdots .
$$

Using the quadratic fitting function $c_{2}\left(g-\bar{g}_{c r}^{(1)}\right)^{2}$, we get the following results

\begin{tabular}{|c|c||c|c||c|c|}
\hline$g_{0}$ & Fit & $g_{0}$ & Fit & $g_{0}$ & Fit \\
\hline 6.2 & $0.0028(g-11.52)^{2}$ & 6.3 & $0.0028(g-11.54)^{2}$ & 6.4 & $0.0028(g-11.55)^{2}$ \\
6.5 & $0.0028(g-11.57)^{2}$ & 6.6 & $0.0028(g-11.59)^{2}$ & 6.7 & $0.0027(g-11.60)^{2}$ \\
6.8 & $0.0027(g-11.62)^{2}$ & 6.9 & $0.0027(g-11.64)^{2}$ & 7. & $0.0027(g-11.65)^{2}$ \\
\hline
\end{tabular}

The results in the table (3.6) are compatible with those in table (3.2). For all values of $g_{0}$ the estimated critical value $g_{c r}^{(1)}$ appears to be less than the corresponding subcritical one from (3.2). They still do not differ much, and it is what one gets from the analysis of asymptotic Y-functions [28].

We conclude from the table (3.6) that $g_{c r}^{(1)}>11.6$, and it seems reasonable to expect that $g_{c r}^{(1)}$ would not exceed 12.0 (it might appear to be a too optimistic expectation), so the first critical value of $\lambda$ would be in the interval $5300<\lambda_{c r}^{(1)}<$ 5700 . 

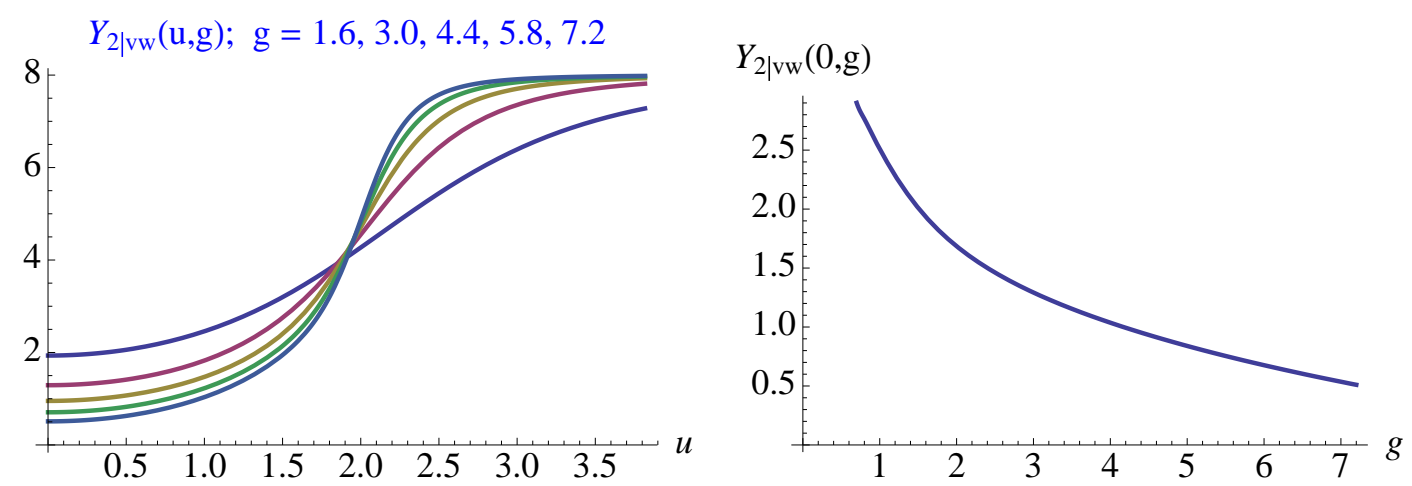

Figure 16: $Y_{2 \mid v w}$-function is approaching 0 at $u=0$ with $g$ increasing. It also approaches its ground state value at $u=\infty$ faster for larger $g$.
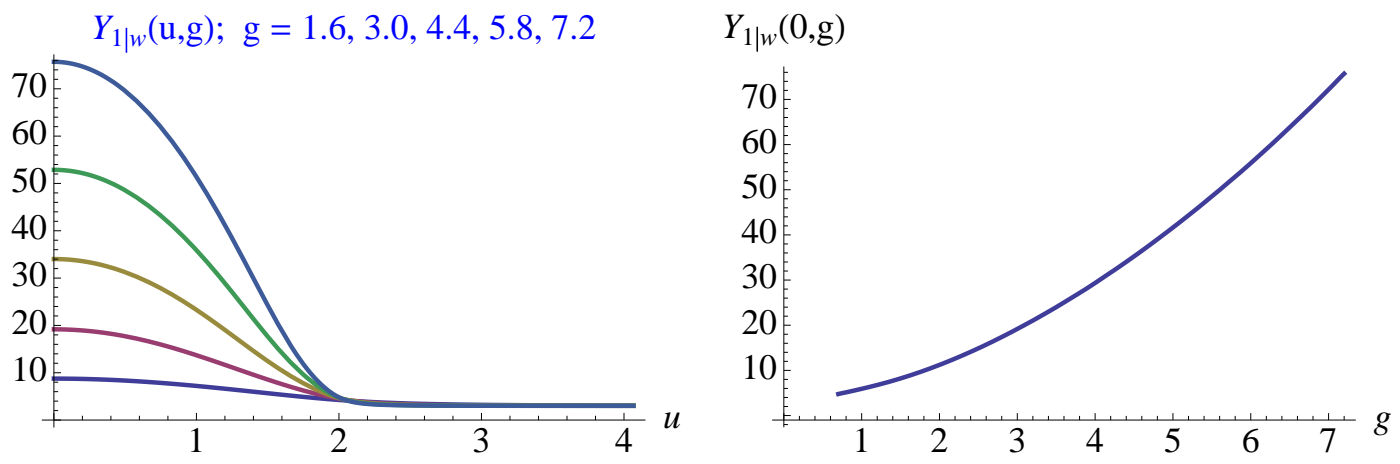

Figure 17: On the left figure graphs of five $Y_{1 \mid w}$-functions with different values of $g$ are shown, and on the right figure the graph of $Y_{1 \mid w}(0, g)$ as a function of $g$ is plotted.

The first subcritical value can be also determined from $Y_{2 \mid v w}$, see Figure 16, because its value at $u=0$ vanishes, and it has the following expansion

$$
Y_{2 \mid v w}(0, g) \sim g-\bar{g}_{c r}^{(1)}+\cdots .
$$

$Y_{2 \mid v w}(0, g)$ is also pretty far from 0 and its linear extrapolation to larger values of $g$ gives results slightly lower than those from table (3.2)

\begin{tabular}{|c|c||c|c||c|c|}
\hline$g_{0}$ & Fit & $g_{0}$ & Fit & $g_{0}$ & Fit \\
\hline 6.2 & $-0.137(g-10.91)$ & 6.3 & $-0.136(g-10.94)$ & 6.4 & $-0.135(g-10.97)$ \\
6.5 & $-0.134(g-11.00)$ & 6.6 & $-0.133(g-11.02)$ & 6.7 & $-0.132(g-11.05)$ \\
6.8 & $-0.131(g-11.08)$ & 6.9 & $-0.130(g-11.10)$ & 7. & $-0.130(g-11.13)$ \\
\hline
\end{tabular}

The estimates can be made closer to the previous ones if one fits the data to higher order polynomials.

Let us finally mention that $Y_{w}$-functions do not show any particular $g$-dependence. They just are increasing very fast, see Figure 17.

It is worth stressing that the estimate of $g_{c r}^{(1)}$ was made under an assumption that the first critical value exists. If it does not then $Y_{1 \mid v w}(0, g)$ would (or not) approach -1 exponentially slow at $g \rightarrow \infty$. 


\section{Large $\lambda$ expansion from the numerical data}

As was discussed in section 2 at large values of 't Hooft's coupling the Konishi state energy admits an asymptotic expansion in powers of $1 / \sqrt[4]{\lambda}$

$$
E_{K}(\lambda)=c_{-1} \sqrt[4]{\lambda}+c_{0}+\frac{c_{1}}{\sqrt[4]{\lambda}}+\frac{c_{2}}{\sqrt{\lambda}}+\frac{c_{3}}{\lambda^{3 / 4}}+\frac{c_{4}}{\lambda}+\frac{c_{5}}{\lambda^{5 / 4}}+\cdots
$$

In this section we try to understand to what extent our numerical data can be used to fix the coefficients $c_{i}$. We should point out however that in general an asymptotic series cannot be found reliably from numerical data. For example, the function $2 \frac{1-e^{1000000-\sqrt{\lambda}}}{1+e^{1000000-\sqrt{\lambda}}} \sqrt[4]{1+\lambda}$ obviously asymptotes to $2 \sqrt[4]{\lambda}$ but any numerical computation performed for $\lambda<1000000$ would predict that it asymptotes to $-2 \sqrt[4]{\lambda}$. Thus, we have to assume first of all that exponentially suppressed terms become very small already at the values of $\lambda$ we are dealing with. Then, a function can approach its asymptotic series monotonically or in oscillations, and it does not seems possible to single out one from numerics. In fact, using the standard least-square fitting procedure would always lead to an oscillating behavior of numerical data about a fitting function. Next, if $\lambda$ is not large enough then one may need to make an assumption about the structure of the large $\lambda$ expansion, for example to decide if the series contains all possible terms or some of them vanish. Finally, fitting numerical data one should decide how many terms one should keep in an asymptotic series, and what fitting interval one should use.

Since the precision of our computation is about $10^{-4}$ for $g \sim 7$ it seems reasonable to keep only the terms up to the $1 / \lambda^{5 / 4}$ order in the asymptotic expansion (4.1). The fitting is done by using the data with the string tension taking values in the interval $\left[g_{0}, g_{1}\right]$ where $g_{1}$ changes from 4.0 to 7.2 (with the step 0.1 ), and Mathematica's Fit (or FindFit) functions. The first point of the fitting interval is chosen to be $g_{0}=1.4$, $\lambda \approx 77$ because it is larger than the inflection point of the Konishi state energy which is $g_{\text {inf }} \approx 0.8, \lambda \approx 25$.

We begin the fitting by making no assumption about the structure of the large $\lambda$ expansion. Below we present the table (4.2) where we use the function in (4.1) to fit our numerical results in (6.1).

\begin{tabular}{|c|c|c|c|}
\hline$g_{1}$ & $\lambda_{1}$ & Fit \\
\hline 4. & 632. & $0.0748723-\frac{19.5546}{\lambda^{5 / 4}}-\frac{26.8658}{\lambda^{3 / 4}}+1.99888 \sqrt[4]{\lambda}+\frac{0.886704}{\sqrt[4]{\lambda}}+\frac{7.23013}{\sqrt{\lambda}}+\frac{40.4154}{\lambda}$ \\
4.4 & 764. & $1.3609-\frac{176.565}{\lambda^{5 / 4}}-\frac{198.245}{\lambda^{3 / 4}}+1.94263 \sqrt[4]{\lambda}-\frac{11.2876}{\sqrt[4]{\lambda}}+\frac{68.3193}{\sqrt{\lambda}}+\frac{295.295}{\lambda}$ \\
4.8 & 910. & $0.94197-\frac{117.655}{\lambda^{5 / 4}}-\frac{137.196}{\lambda^{3 / 4}}+1.96036 \sqrt[4]{\lambda}-\frac{7.19535}{\sqrt[4]{\lambda}}+\frac{47.1638}{\sqrt{\lambda}}+\frac{202.045}{\lambda}$ \\
5.2 & 1070. & $0.443739-\frac{41.3538}{\lambda^{5 / 4}}-\frac{60.5348}{\lambda^{3 / 4}}+1.98102 \sqrt[4]{\lambda}-\frac{2.23404}{\sqrt[4]{\lambda}}+\frac{21.0468}{\sqrt{\lambda}}+\frac{83.0606}{\lambda}$ \\
5.6 & 1240. & $-0.0777338+\frac{45.6551}{\lambda^{5 / 4}}+\frac{24.2251}{\lambda^{3 / 4}}+2.0022 \sqrt[4]{\lambda}+\frac{3.06072}{\sqrt[4]{\lambda}}-\frac{7.33942}{\sqrt{\lambda}}-\frac{50.6135}{\lambda}$ \\
6. & 1420. & $-0.439602+\frac{110.23}{\lambda^{5 / 4}}+\frac{85.6348}{\lambda^{3 / 4}}+2.01667 \sqrt[4]{\lambda}+\frac{6.79178}{\sqrt[4]{\lambda}}-\frac{27.6317}{\sqrt{\lambda}}-\frac{148.678}{\lambda}$ \\
6.4 & 1620. & $-0.131008+\frac{51.482}{\lambda^{5 / 4}}+\frac{31.0414}{\lambda^{3 / 4}}+2.00453 \sqrt[4]{\lambda}+\frac{3.56246}{\sqrt[4]{\lambda}}-\frac{9.8231}{\sqrt{\lambda}}-\frac{60.4474}{\lambda}$ \\
6.8 & 1830. & $0.201473-\frac{15.5603}{\lambda^{5 / 4}}-\frac{30.0019}{\lambda^{3 / 4}}+1.99163 \sqrt[4]{\lambda}+\frac{0.0366606}{\sqrt[4]{\lambda}}+\frac{9.86239}{\sqrt{\lambda}}+\frac{39.2596}{\lambda}$ \\
7.2 & 2050. & $0.210769-\frac{17.5595}{\lambda^{5 / 4}}-\frac{31.7814}{\lambda^{3 / 4}}+1.99128 \sqrt[4]{\lambda}-\frac{0.0634158}{\sqrt[4]{\lambda}}+\frac{10.429}{\sqrt{\lambda}}+\frac{42.2007}{\lambda}$ \\
\hline
\end{tabular}



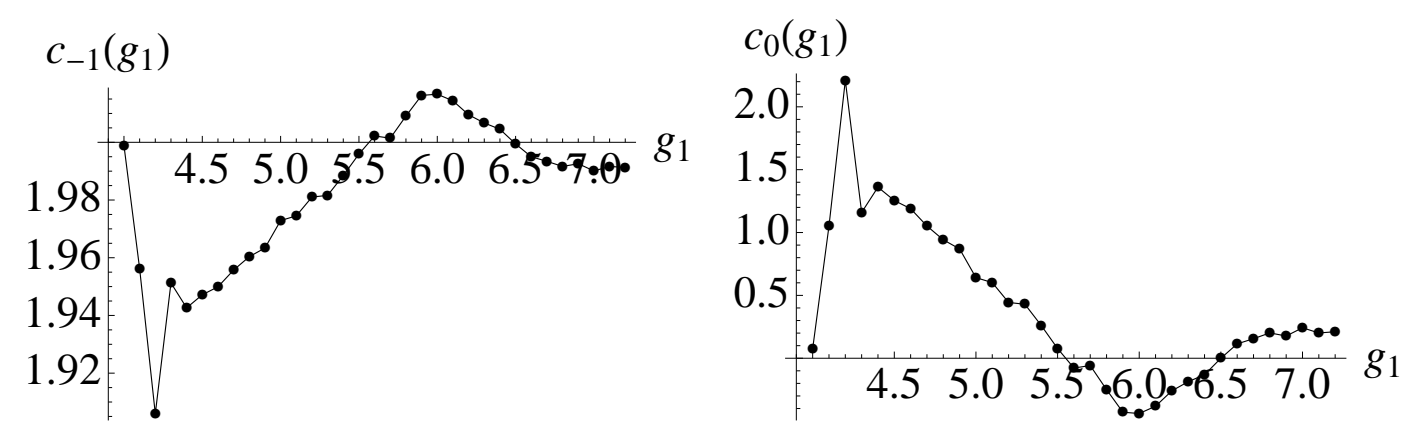

Figure 18: On the left and right figures the graphs of $c_{-1}$ and $c_{0}$ as functions of $g_{1}$ are plotted. The fitting is done without any constraint imposed on $c_{i}$.

To better visualize the results in Figure 18 we also plot the coefficients $c_{-1}$ and $c_{0}$. As one can see, the coefficient $c_{-1}$ of the leading term is oscillating about 2 , and for $g_{1}>6.2$ it is getting quite close to 2 . Its average value is 1.983 . The coefficient $c_{0}$ is oscillating about 0 but it is not really small with the average equal to 0.385 . There is however an obvious correlation between the values of $c_{-1}$ and $c_{0}$ - the closer $c_{-1}$ is to 2 the closer $c_{0}$ is to 0 .

The subleading coefficients are however not fixed at all and take very different values depending on the fitting interval used. The fitting therefore is not stable, and the fitting function strongly depends on the fitting interval. One may conclude that the numerical data allows one to fix only the leading coefficient in the strong coupling expansion. One should remember however that the series (4.1) is only asymptotic, and fixing its coefficients by using the data for these not very large values of $\lambda$ is not that straightforward as it is for a convergent series.

To proceed let us fix the leading coefficient to be 2 . Then, fitting the numerical data, one finds the results in table (4.3) and Figure 19.

$$
\begin{array}{|c|c|c|c|}
\hline g_{1} & \lambda_{1} & \text { Fit } \\
\hline 4 . & 632 . & 0.0487754-\frac{16.0827}{\lambda^{5 / 4}}-\frac{23.1977}{\lambda^{3 / 4}}+2 \sqrt[4]{\lambda}+\frac{1.13832}{\sqrt[4]{\lambda}}+\frac{5.94492}{\sqrt{\lambda}}+\frac{34.8688}{\lambda} \\
4.4 & 764 . & -0.00945538+\frac{26.2666}{\lambda^{5 / 4}}+\frac{7.84673}{\lambda^{3 / 4}}+2 \sqrt[4]{\lambda}+\frac{2.2426}{\sqrt[4]{\lambda}}-\frac{2.36707}{\sqrt{\lambda}}-\frac{22.6721}{\lambda} \\
4.8 & 910 . & -0.0269174+\frac{40.3386}{\lambda^{5 / 4}}+\frac{17.7895}{\lambda^{3 / 4}}+2 \sqrt[4]{\lambda}+\frac{2.58159}{\sqrt[4]{\lambda}}-\frac{4.97525}{\sqrt{\lambda}}-\frac{41.4559}{\lambda} \\
5.2 & 1070 . & -0.030125+\frac{43.0799}{\lambda^{5 / 4}}+\frac{19.6865}{\lambda^{3 / 4}}+2 \sqrt[4]{\lambda}+\frac{2.64471}{\sqrt[4]{\lambda}}-\frac{5.46707}{\sqrt{\lambda}}-\frac{45.0787}{\lambda} \\
5.6 & 1240 . & -0.0216031+\frac{34.8027}{\lambda^{5 / 4}}+\frac{14.2113}{\lambda^{3 / 4}}+2 \sqrt[4]{\lambda}+\frac{2.47195}{\sqrt[4]{\lambda}}-\frac{4.08328}{\sqrt{\lambda}}-\frac{34.3737}{\lambda} \\
6 . & 1420 . & -0.00723624+\frac{20.0648}{\lambda^{5 / 4}}+\frac{4.64678}{\lambda^{3 / 4}}+2 \sqrt[4]{\lambda}+\frac{2.17693}{\sqrt[4]{\lambda}}-\frac{1.69214}{\sqrt{\lambda}}-\frac{15.4861}{\lambda} \\
6.4 & 1620 . & -0.0115075+\frac{24.7422}{\lambda^{5 / 4}}+\frac{7.61427}{\lambda^{3 / 4}}+2 \sqrt[4]{\lambda}+\frac{2.266}{\sqrt[4]{\lambda}}-\frac{2.42443}{\sqrt{\lambda}}-\frac{21.4161}{\lambda} \\
6.8 & 1830 . & -0.0230903+\frac{38.1119}{\lambda^{5 / 4}}+\frac{15.9437}{\lambda^{3 / 4}}+2 \sqrt[4]{\lambda}+\frac{2.5106}{\sqrt[4]{\lambda}}-\frac{4.45848}{\sqrt{\lambda}}-\frac{38.2204}{\lambda} \\
7.2 & 2050 . & -0.0269859+\frac{42.8933}{\lambda^{5 / 4}}+\frac{18.8607}{\lambda^{3 / 4}}+2 \sqrt[4]{\lambda}+\frac{2.59408}{\sqrt[4]{\lambda}}-\frac{5.16215}{\sqrt{\lambda}}-\frac{44.1706}{\lambda} \\
\hline
\end{array}
$$

The coefficient $c_{0}$ is now much smaller but it stops oscillating about 0 . The average value of $c_{0}$ is -0.016 , and $c_{0}$ is decreasing with $g_{1}$ increasing. The subleading coefficient $c_{1}$ is not close to 2 , it has an average 2.37, and is greater than 2 for almost all values of $g_{1}$. There is still a correlation between the values of $c_{0}$ and $c_{1}$ - the closer 


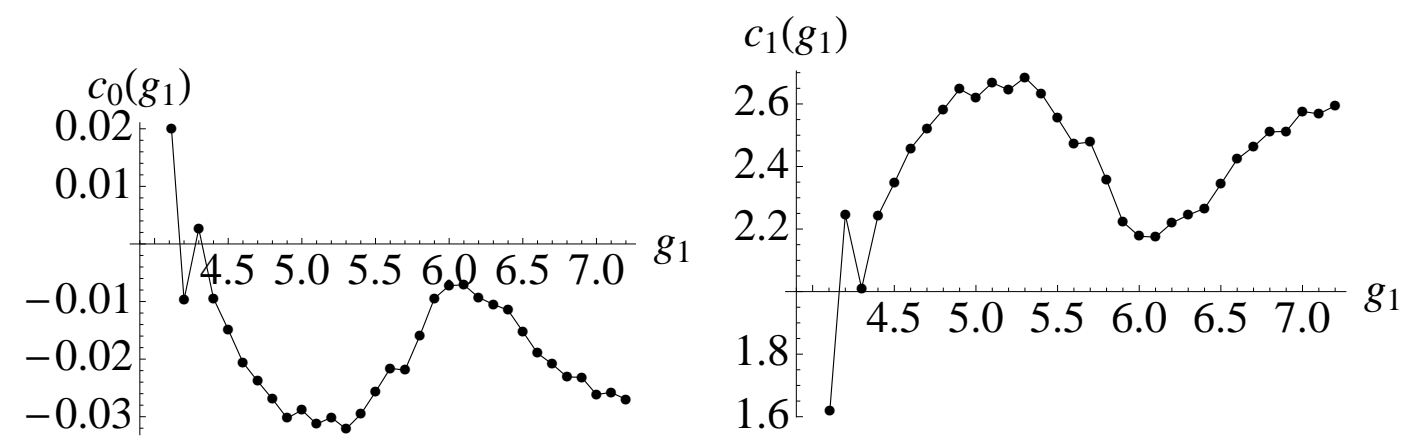

Figure 19: On the left and right figures the graphs of $c_{0}$ and $c_{1}$ as functions of $g_{1}$ are plotted. The fitting is done with $c_{-1}=2$.

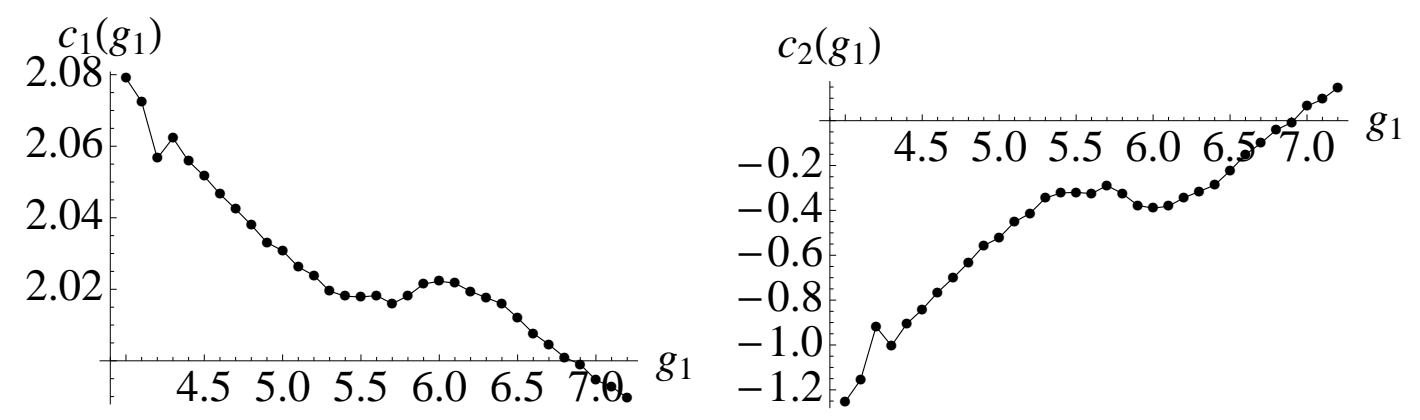

Figure 20: On the left and right figures the graphs of $c_{1}$ and $c_{2}$ as functions of $g_{1}$ are plotted. The fitting is done with $c_{-1}=2$ and $c_{0}=0$.

$c_{0}$ is to 0 the closer $c_{1}$ is to 2 . One sees that for the range of $\lambda$ we are analyzing the contribution of the constant term is much smaller than the one of the subleading term $c_{1} / \sqrt[4]{\lambda}$ and, therefore, it is reasonable to assume that $c_{0}=0$.

Fixing now the leading and constant coefficients to be 2 and 0 , respectively, one finds the results in table (4.4) and Figure 20.

\begin{tabular}{|c|c|c|}
\hline$g_{1}$ & $\lambda_{1}$ & Fit \\
\hline 4. & 632. & $\frac{22.2908}{\lambda^{5 / 4}}+\frac{4.1118}{\lambda^{3 / 4}}+2 \sqrt[4]{\lambda}+\frac{2.07902}{\sqrt[4]{\lambda}}-\frac{1.25263}{\sqrt{\lambda}}-\frac{16.5214}{\lambda}$ \\
4.4 & 764. & $\frac{18.0024}{\lambda^{5 / 4}}+\frac{2.18271}{\lambda^{3 / 4}}+2 \sqrt[4]{\lambda}+\frac{2.0558}{\sqrt[4]{\lambda}}-\frac{0.905321}{\sqrt{\lambda}}-\frac{11.8034}{\lambda}$ \\
4.8 & 910. & $\frac{14.458}{\lambda^{5 / 4}}+\frac{0.641502}{\lambda^{3 / 4}}+2 \sqrt[4]{\lambda}+\frac{2.03799}{\sqrt[4]{\lambda}}-\frac{0.633195}{\sqrt{\lambda}}-\frac{7.96653}{\lambda}$ \\
5.2 & 1070. & $\frac{11.4777}{\lambda^{5 / 4}}-\frac{0.621309}{\lambda^{3 / 4}}+2 \sqrt[4]{\lambda}+\frac{2.02384}{\sqrt[4]{\lambda}}-\frac{0.413548}{\sqrt{\lambda}}-\frac{4.77967}{\lambda}$ \\
5.6 & 1240. & $\frac{10.2483}{\lambda^{5 / 4}}-\frac{1.13316}{\lambda^{3 / 4}}+2 \sqrt[4]{\lambda}+\frac{2.01824}{\sqrt[4]{\lambda}}-\frac{0.325432}{\sqrt{\lambda}}-\frac{3.47594}{\lambda}$ \\
6. & 1420. & $\frac{11.2064}{\lambda^{5 / 4}}-\frac{0.748872}{\lambda^{3 / 4}}+2 \sqrt[4]{\lambda}+\frac{2.02225}{\sqrt[4]{\lambda}}-\frac{0.390144}{\sqrt{\lambda}}-\frac{4.47424}{\lambda}$ \\
6.4 & 1620. & $\frac{9.64851}{\lambda^{5 / 4}}-\frac{1.36445}{\lambda^{3 / 4}}+2 \sqrt[4]{\lambda}+\frac{2.01594}{\sqrt[4]{\lambda}}-\frac{0.287401}{\sqrt{\lambda}}-\frac{2.86239}{\lambda}$ \\
6.8 & 1830. & $\frac{5.80801}{\lambda^{5 / 4}}-\frac{2.85568}{\lambda^{3 / 4}}+2 \sqrt[4]{\lambda}+\frac{2.00097}{\sqrt[4]{\lambda}}-\frac{0.041104}{\sqrt{\lambda}}+\frac{1.07875}{\lambda}$ \\
7.2 & 2050. & $\frac{2.78511}{\lambda^{5 / 4}}-\frac{4.00844}{\lambda^{3 / 4}}+2 \sqrt[4]{\lambda}+\frac{1.98967}{\sqrt[4]{\lambda}}+\frac{0.147219}{\sqrt{\lambda}}+\frac{4.15477}{\lambda}$ \\
\hline
\end{tabular}

It is clear from the table and Figure 20 that fixing $c_{-1}=2$ and $c_{0}=0$ makes the first nontrivial subleading coefficient $c_{1}$ to be very close to 2 . Its average value is $\approx 2.026$. The next coefficient $c_{2}$ still varies significantly and its average is -0.435 . 

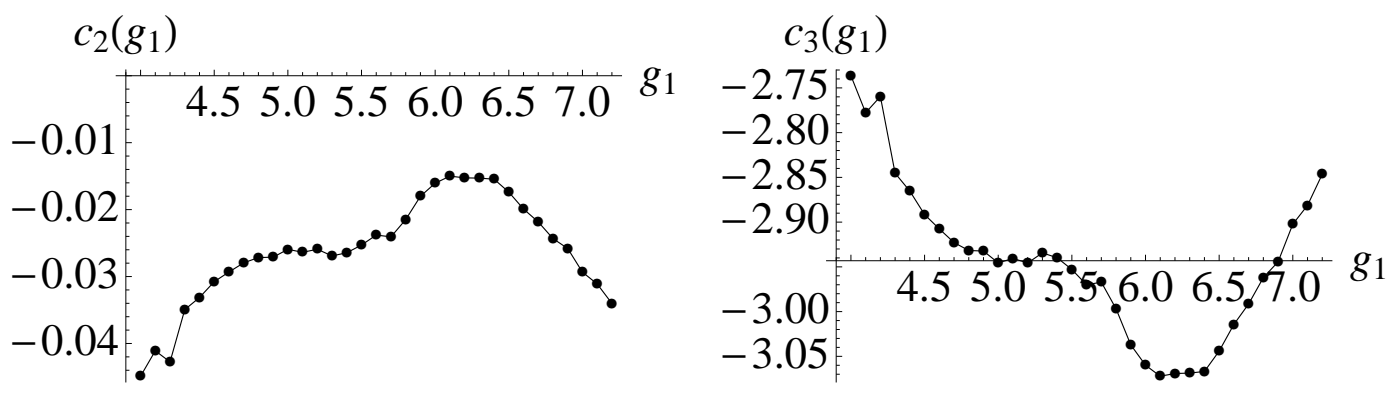

Figure 21: On the left and right figures the graphs of $c_{2}$ and $c_{3}$ as functions of $g_{1}$ are plotted. The fitting is done with $c_{-1}=2, c_{0}=0$ and $c_{1}=2$.

However $c_{2}$ is increasing and becomes very close to 0 for $g_{1}=6.9$.

Since the coefficient $c_{1}$ is so close to 2 , let us proceed by fixing $c_{-1}=2, c_{0}=0$, and $c_{1}=2$. Then, one obtains the following table and Figure 21

\begin{tabular}{|c|c|c|}
\hline$g_{1}$ & $\lambda_{1}$ & Fit \\
\hline 4. & 632. & $\frac{6.48139}{1 \mathrm{a}^{5 / 4}}-\frac{2.7364}{1 \mathrm{a}^{3 / 4}}+2 \sqrt[4]{\mathrm{la}}+\frac{2}{\sqrt[4]{\mathrm{la}}}-\frac{0.044888}{\sqrt{\mathrm{la}}}+\frac{0.556775}{\mathrm{la}}$ \\
4.4 & 764. & $\frac{5.92711}{1 \mathrm{a}^{5 / 4}}-\frac{2.86533}{1 \mathrm{a}^{3 / 4}}+2 \sqrt[4]{\mathrm{la}}+\frac{2}{\sqrt[4]{\mathrm{la}}}-\frac{0.0331335}{\sqrt{\mathrm{la}}}+\frac{1.02244}{\mathrm{la}}$ \\
4.8 & 910. & $\frac{5.63528}{1 \mathrm{a}^{5 / 4}}-\frac{2.93171}{1 \mathrm{a}^{3 / 4}}+2 \sqrt[4]{\mathrm{la}}+\frac{2}{\sqrt[4]{\mathrm{la}}}-\frac{0.0271635}{\sqrt{\mathrm{la}}}+\frac{1.26505}{\mathrm{la}}$ \\
5.2 & 1070. & $\frac{5.57229}{1 \mathrm{a}^{5 / 4}}-\frac{2.94569}{1 \mathrm{a}^{3 / 4}}+2 \sqrt[4]{\mathrm{la}}+\frac{2}{\sqrt[4]{\mathrm{la}}}-\frac{0.0259249}{\sqrt{\mathrm{la}}}+\frac{1.31682}{\mathrm{la}}$ \\
5.6 & 1240. & $\frac{5.45878}{1 \mathrm{a}^{5 / 4}}-\frac{2.97011}{1 \mathrm{a}^{3 / 4}}+2 \sqrt[4]{\mathrm{la}}+\frac{2}{\sqrt[4]{\mathrm{la}}}-\frac{0.0238023}{\sqrt{\mathrm{la}}}+\frac{1.40878}{\mathrm{la}}$ \\
6. & 1420. & $\frac{5.03661}{1 \mathrm{a}^{5 / 4}}-\frac{3.0599}{1 \mathrm{a}^{3 / 4}}+2 \sqrt[4]{\mathrm{la}}+\frac{2}{\sqrt[4]{\mathrm{la}}}-\frac{0.0160542}{\sqrt{\mathrm{la}}}+\frac{1.749}{\mathrm{a}}$ \\
6.4 & 1620. & $\frac{5.00365}{1 \mathrm{a}^{5 / 4}}-\frac{3.06689}{1 \mathrm{a}^{3 / 4}}+2 \sqrt[4]{\mathrm{la}}+\frac{2}{\sqrt[4]{\mathrm{la}}}-\frac{0.0154523}{\sqrt{\mathrm{la}}}+\frac{1.77553}{\mathrm{la}}$ \\
6.8 & 1830. & $\frac{5.51203}{1 \mathrm{a}^{5 / 4}}-\frac{2.96201}{1 \mathrm{a}^{3 / 4}}+2 \sqrt[4]{\mathrm{la}}+\frac{2}{\sqrt[4]{\mathrm{la}}}-\frac{0.0243275}{\sqrt{\mathrm{la}}}+\frac{1.37154}{\mathrm{la}}$ \\
7.2 & 2050. & $\frac{6.08044}{1 \mathrm{a}^{5 / 4}}-\frac{2.84643}{1 \mathrm{l}^{3 / 4}}+2 \sqrt[4]{\mathrm{la}}+\frac{2}{\sqrt[4]{\mathrm{la}}}-\frac{0.0340167}{\sqrt{\mathrm{la}}}+\frac{0.922834}{\mathrm{la}}$ \\
\hline
\end{tabular}

The fitting with the subleading coefficient equal to 2 makes the coefficient $c_{2}$ to be very small with the average -0.026 . Its contribution to the energy is much smaller than the contribution of the next term $c_{3} / \lambda^{3 / 4}$, and it is reasonable to assume that $c_{2}$ is equal to 0 . So, let us proceed by fixing $c_{-1}=2, c_{0}=0, c_{1}=2$, and $c_{2}=0$. Then, one obtains the following table and Figure 22

\begin{tabular}{|c|c|c|}
\hline$g_{1}$ & $\lambda_{1}$ & Fit \\
\hline 4. & 632. & $\frac{4.19637}{1 a^{5 / 4}}-\frac{3.24272}{1 a^{3 / 4}}+2 \sqrt[4]{\mathrm{la}}+\frac{2}{\sqrt[4]{\mathrm{la}}}+\frac{2.43269}{\mathrm{la}}$ \\
\hline 4.4 & 764. & $\frac{4.15138}{1 \mathrm{a}^{5 / 4}}-\frac{3.24647}{\mathrm{la}^{3 / 4}}+2$ \\
\hline 4.8 & 910. & $\frac{4.11145}{\mathrm{l}^{5 / 4}}-\frac{3.24973}{\mathrm{a}^{3 / 4}}+2 \sqrt[4]{\mathrm{la}}+\frac{2}{\sqrt[4]{\mathrm{la}}}+\frac{2.48182}{\mathrm{la}}$ \\
\hline 5.2 & 1070. & $\frac{4.05719}{1 a^{5 / 4}}-\frac{3.25408}{l a^{3 / 4}}+2 \sqrt[4]{l a}+$ \\
\hline 5.6 & 1240. & $\frac{4.0154}{10^{5 / 4}}-\frac{3.2574}{l^{3 / 4}}$ \\
\hline 6 & 1420. & $\frac{4.02985}{l a^{5 / 4}}-\frac{3.25628}{l a^{3 / 4}}+$ \\
\hline 6.4 & 1620 . & $\frac{4.00445}{1 \mathrm{a}^{5 / 4}}-\frac{3.25825}{\mathrm{l}^{3 / 4}}$ \\
\hline 6.8 & 1830. & $\frac{3.89399}{l^{5 / 4}}-\frac{3.26672}{l^{3 / 4}}$ \\
\hline 7.2 & 2050. & $\frac{3.75839}{1 \mathrm{a}^{5 / 4}}-\frac{3.27702}{1 \mathrm{a}^{3 / 4}}+$ \\
\hline
\end{tabular}

We see an interesting effect of this fitting. It appears to be rather stable. As one can see from the table (4.5), the remaining three coefficients $c_{3}, c_{4}$ and $c_{5}$ are not in fact too sensitive to the choice of $g_{1}$ anymore. 


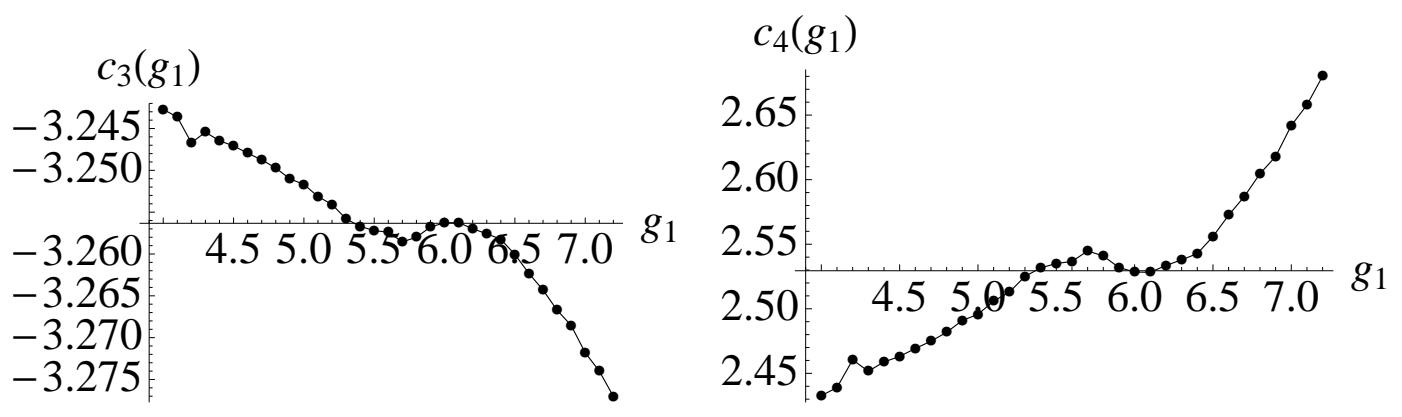

Figure 22: On the left and right figures the graphs of $c_{2}$ and $c_{3}$ as functions of $g_{1}$ are plotted. The fitting is done with $c_{-1}=2, c_{0}=0$ and $c_{1}=2$.

To continue we use the average value of $c_{3}$, and fit the coefficients $c_{4}$ and $c_{5}$. Then, we use the average value of $c_{4}$, and fit the coefficient $c_{5}$. Finally, taking the average value of $c_{5}$, we find the following fitting function

$$
g_{0}=1.4: \quad \bar{E}_{K}(\lambda)=2 \sqrt[4]{\lambda}+\frac{2}{\sqrt[4]{\lambda}}-\frac{3.26}{\lambda^{3 / 4}}+\frac{2.53}{\lambda}+\frac{4.03}{\lambda^{5 / 4}} .
$$

Obviously, the fitting function is different from (1.1), in particular the coefficient $c_{4}$ is not small and gives a significant contribution to the energy. The coefficients $c_{i}$ in (4.7) depend on the choice of $g_{0}$. In the table below we present fitting functions for $1.2 \leq g_{0} \leq 1.6$

$$
\begin{array}{|c|c|}
\hline g_{0} & \bar{E}_{K} \\
\hline 1.2 & 2 \sqrt[4]{\lambda}+\frac{2}{\sqrt[4]{\lambda}}-\frac{3.18}{\lambda^{3 / 4}}+\frac{1.89}{\lambda}+\frac{5.27}{\lambda^{5 / 4}} \\
1.3 & 2 \sqrt[4]{\lambda}+\frac{2}{\sqrt[4]{\lambda}}-\frac{3.22}{\lambda^{3 / 4}}+\frac{2.25}{\lambda}+\frac{4.57}{\lambda^{5 / 4}} \\
1.4 & 2 \sqrt[4]{\lambda}+\frac{2}{\sqrt[4]{\lambda}}-\frac{3.26}{\lambda^{3 / 4}}+\frac{2.53}{\lambda}+\frac{4.03}{\lambda^{5 / 4}} \\
1.5 & 2 \sqrt[4]{\lambda}+\frac{2}{\sqrt[4]{\lambda}}-\frac{3.27}{\lambda^{3 / 4}}+\frac{2.63}{\lambda}+\frac{3.83}{\lambda^{5 / 4}} \\
1.6 & 2 \sqrt[4]{\lambda}+\frac{2}{\sqrt[4]{\lambda}}-\frac{3.28}{\lambda^{3 / 4}}+\frac{2.70}{\lambda}+\frac{3.69}{\lambda^{5 / 4}} \\
\hline
\end{array}
$$

One can see that only $c_{3}$ is relatively stable $c_{3}=-3.23 \pm 0.05$ but the other coefficients change more substantially.

Does our fitting rule out the strong coupling expansion in powers of $1 / \sqrt{\lambda}$ advocated in [29]? In Figure 23 we compare the fitting function $\bar{E}_{K}(\lambda)(4.7)$ with two fitting functions obtained by setting $c_{2 k}=0$ from the very beginning. Both $\bar{E}_{K}(\lambda)$ and the function (1.3) fit the data equally well. This is not surprising because both functions have the same number of free fitting parameters. On the other hand, the function $\bar{E}_{K}^{\mathrm{GKV}}(\lambda)=2 \sqrt[4]{\lambda}+\frac{2}{\sqrt[4]{\lambda}}-\frac{2.94}{\lambda^{3 / 4}}+\frac{8.83}{\lambda^{5 / 4}}$ obtained by setting $c_{-1}=c_{1}=2$ and $c_{2 k}=0$ significantly deviates from the data, up to 0.0002 , which is at least twice more than the precision of our computation for $g<5$. Thus, we are to conclude that the coefficient $c_{4}$ does not vanish and the strong coupling expansion is in powers of $1 / \sqrt[4]{\lambda}$. Having said this, we should admit that if the actual precision of our 

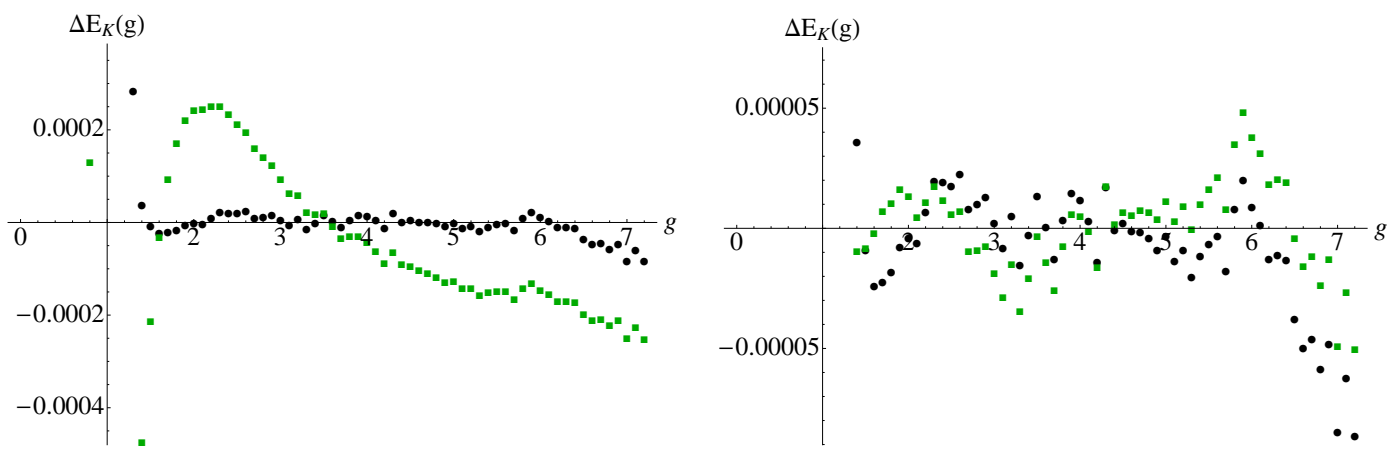

Figure 23: Black dots represent the difference between the computed values of the Konishi energy and its fitting function (4.7): $\Delta E_{K}=E_{K}-\bar{E}_{K}$. Green squares on the left picture represent a similar difference with $\bar{E}_{K}^{\text {GKV }}$ where the fitting function $\bar{E}_{K}^{\mathrm{GKV}}(\lambda)=2 \sqrt[4]{\lambda}+\frac{2}{\sqrt[4]{\lambda}}-\frac{2.94}{\lambda^{3 / 4}}+\frac{8.83}{\lambda^{5 / 4}}$ is obtained by using our data and setting $c_{-1}=c_{1}=2$ and $c_{0}=c_{2}=c_{4}=0$. On the right picture green squares represent the difference of $E_{K}$ and the fitting function in (1.2).
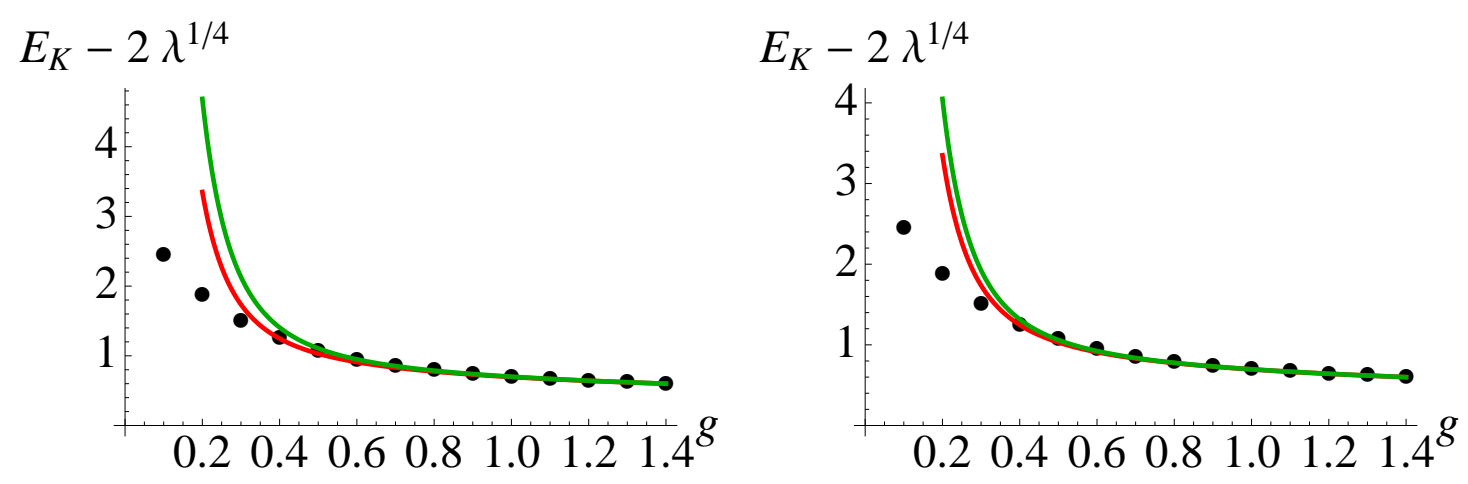

Figure 24: Black dots represent the difference between the energy and its leading large $\lambda$ asymptotic, $E_{K}-2 \sqrt[4]{\lambda}$. Red (upper) curve is the graph of $\bar{E}_{K}-2 \sqrt[4]{\lambda}$. Green curve on the left picture is the graph of $\bar{E}_{K}^{\mathrm{GKV}}(\lambda)-2 \sqrt[4]{\lambda}$, and on the right picture it is the difference between the fitting function (1.3) and $2 \sqrt[4]{\lambda}$. The red curve works very well starting already with $g=0.3, \lambda=3.55$.

computation for $g \sim 7.0$ is not 0.0001 but about 0.0002 , and if the contribution of exponentially suppressed terms is of order 0.0002 for $g \sim 2$, then this could explain the left plot on Figure 23 and make possible the vanishing of $c_{4}$.

Let us finally mention that the function $\bar{E}_{K}$ works unexpectedly well (and better than $\bar{E}_{K}^{\mathrm{GKV}}$ or (1.3)) starting already with such a small value of the coupling constant as $g=0.3, \lambda=3.55$, see Figure 24, that is less than the expected radius of convergency of the weak-coupling expansion, even though the fitting was done for the data with $g \geq 1.4$. This implicitly confirms that $c_{1}=2$. Since $\sqrt[4]{3.55}=1.37$ is close to 1 , changing $c_{1}$ just by 0.1 would require an essential change of the coefficients $c_{3}, c_{4}$ and $c_{5}$ to fit the energy data at large $\lambda$ but then the fitting at small $\lambda$ would be destroyed.

In Figure 25 we compare our numerical results with those of [29]. 


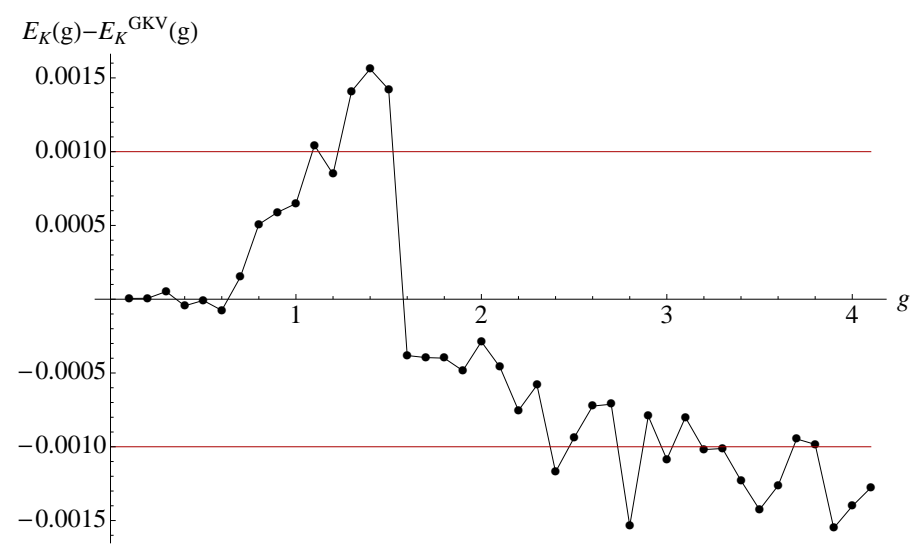

Figure 25: The black dots represent the difference between the values of the Konishi energy we obtained and those from [29]. One sees that our results agree with those of [29] with 0.0015 precision for almost all values of $g$.

\section{Conclusion}

In this paper we solved the TBA equations for the Konishi operator descendent from the $\mathfrak{s l}(2)$ sector proposed by Arutyunov, Suzuki and the author [28] up to 't Hooft's coupling $\lambda \approx 2046$. At this value the iterations converge very slowly. It would be important to improve the numerical algorithm and approach closer to the first critical value of $\lambda$, and go beyond it. One possible improvement would be to use Newton's method for solving the TBA equations as it was done for the Hubbard model in [65]. Solving the TBA equations numerically for large values of $\lambda$ is a challenging problem also because at large $\lambda$ the Konishi state energy is very close to the energy of the other two Konishi-like states with $n=1$, see [28] for detail. It would be interesting to repeat the computation for these states, and for other Konishi-like states with larger string levels.

Fitting the data for the Konishi state energy ${ }^{9}$ we found convincingly that the first nontrivial subleading coefficient $c_{1}$ is equal to 2 and the coefficient $c_{2}$ vanishes in agreement with the previous prediction [29]. The coefficient $c_{4}$ however does not vanish and therefore the strong coupling expansion seems to be not in powers of $1 / \sqrt{\lambda}$ (up to an overall $\sqrt[4]{\lambda}$ ) but in powers of $1 / \sqrt[4]{\lambda}$.

In principle increasing the values of $\lambda$ may change the fitting coefficients and invalidate the predictions for the coefficients. It is necessary to derive them by analytic means.

Extrapolating the results obtained shows that the first critical value for the Konishi operator is $\lambda_{c r}^{(1)}>5300$ and it is probably in the range $5300<\lambda_{c r}^{(1)}<5700$ which is significantly higher than the estimate based on the large $J$ asymptotic

\footnotetext{
${ }^{9}$ We assumed the absence of $\log \lambda$-dependent terms in the asymptotic large $\lambda$ expansion. These terms are known to appear in the strong coupling expansion of the Bethe-Yang equations [66, 67].
} 
solution that gives $\lambda_{c r}^{(1)} \approx 774$. This means that even at such a large value of $\lambda$ we are still far from the strong coupling regime. This is an intermediate coupling regime and in fact it is the one where $Y_{Q}$-functions play the most important role. Moreover, if the contribution of $Y_{Q^{-}}$functions to the Konishi state energy will continue growing as $\sqrt{\lambda}$ then this would imply that at large $\lambda$ the exact Bethe root asymptotes to a constant less than 2, and the critical values for the Konishi operator are absent.

It is clear that the numerical computation we performed raised more questions than gave answers. Some of the questions can be answered only analytically, and we hope to address them in future.

\section{Acknowledgements}

The author thanks Gleb Arutyunov, Zoltan Bajnok, Niklas Beisert, Davide Fioravanti, Tristan McLoughlin, Jan Plefka, Radu Roiban, Matthias Staudacher, Ryo Suzuki, Roberto Tateo, Arkady Tseytlin and Kostya Zarembo for interesting discussions and comments on the manuscript, and Dmitri Grigoriev for computer help. This work was supported in part by the Science Foundation Ireland under Grants No. 07/RFP/PHYF104 and 09/RFP/PHY2142, and by a one-month Max-PlanckInstitut für Gravitationsphysik Albert-Einstein-Institut grant.

\section{Appendix}

\section{Numerical data}

Here we collect our numerical data.

In the table (6.1) we present the results of the computation of the energy of the Konishi state or, equivalently, the conformal dimension of the Konishi operator as a function of $g$

\begin{tabular}{|c|c||c|c||c|c||c|c||c|c||c|c|}
\hline$g$ & $\mathrm{E}_{\mathrm{K}}$ & $g$ & $\mathrm{E}_{\mathrm{K}}$ & $g$ & $\mathrm{E}_{\mathrm{K}}$ & $g$ & $\mathrm{E}_{\mathrm{K}}$ & $g$ & $\mathrm{E}_{\mathrm{K}}$ & $g$ & $\mathrm{E}_{\mathrm{K}}$ \\
\hline 0.1 & 4.02971 & 0.2 & 4.11551 & 0.3 & 4.24885 & 0.4 & 4.41886 & 0.5 & 4.61469 & 0.6 & 4.82682 \\
0.7 & 5.04775 & 0.8 & 5.27151 & 0.9 & 5.49399 & 1. & 5.71265 & 1.1 & 5.92614 & 1.2 & 6.13385 \\
1.3 & 6.33561 & 1.4 & 6.53156 & 1.5 & 6.72212 & 1.6 & 6.90752 & 1.7 & 7.0881 & 1.8 & 7.2642 \\
1.9 & 7.43612 & 2. & 7.60411 & 2.1 & 7.76844 & 2.2 & 7.92935 & 2.3 & 8.08702 & 2.4 & 8.24163 \\
2.5 & 8.39336 & 2.6 & 8.54238 & 2.7 & 8.68879 & 2.8 & 8.83276 & 2.9 & 8.97441 & 3. & 9.11381 \\
3.1 & 9.2511 & 3.2 & 9.38638 & 3.3 & 9.51969 & 3.4 & 9.65117 & 3.5 & 9.78087 & 3.6 & 9.90884 \\
3.7 & 10.0352 & 3.8 & 10.1599 & 3.9 & 10.2831 & 4. & 10.4049 & 4.1 & 10.5252 & 4.2 & 10.6442 \\
4.3 & 10.7618 & 4.4 & 10.8782 & 4.5 & 10.9933 & 4.6 & 11.1072 & 4.7 & 11.22 & 4.8 & 11.3316 \\
4.9 & 11.4421 & 5. & 11.5516 & 5.1 & 11.66 & 5.2 & 11.7675 & 5.3 & 11.8739 & 5.4 & 11.9794 \\
5.5 & 12.084 & 5.6 & 12.1877 & 5.7 & 12.2905 & 5.8 & 12.3924 & 5.9 & 12.4936 & 6.4 & 12.5938 \\
6.1 & 12.6933 & 6.2 & 12.792 & 6.3 & 12.89 & 6.4 & 12.9872 & 6.5 & 13.0837 & 6.6 & 13.1795 \\
6.7 & 13.2746 & 6.8 & 13.369 & 6.9 & 13.4627 & 7. & 13.5558 & 7.1 & 13.6483 & 7.2 & 13.7401 \\
\hline
\end{tabular}


In the table (6.2) we present the results of the computation of the Bethe root $w$

\begin{tabular}{|c|c||c|c||c|c||c|c||c|c||c|c|}
\hline$g$ & $w_{K}$ & $g$ & $w_{K}$ & $g$ & $w_{K}$ & $g$ & $w_{K}$ & $g$ & $w_{K}$ & $g$ & $w_{K}$ \\
\hline 0.1 & 5.88827 & 0.2 & 3.11236 & 0.3 & 2.25445 & 0.4 & 1.87021 & 0.5 & 1.67116 & 0.6 & 1.56174 \\
0.7 & 1.50066 & 0.8 & 1.46819 & 0.9 & 1.45317 & 1. & 1.4489 & 1.1 & 1.45121 & 1.2 & 1.45758 \\
1.3 & 1.46638 & 1.4 & 1.47661 & 1.5 & 1.48748 & 1.6 & 1.49866 & 1.7 & 1.50987 & 1.8 & 1.52093 \\
1.9 & 1.53174 & 2. & 1.54224 & 2.1 & 1.55239 & 2.2 & 1.56217 & 2.3 & 1.57159 & 2.4 & 1.58064 \\
2.5 & 1.58934 & 2.6 & 1.59769 & 2.7 & 1.60569 & 2.8 & 1.61339 & 2.9 & 1.62078 & 3. & 1.62787 \\
3.1 & 1.6347 & 3.2 & 1.64127 & 3.3 & 1.64758 & 3.4 & 1.65367 & 3.5 & 1.65953 & 3.6 & 1.66517 \\
3.7 & 1.67063 & 3.8 & 1.67588 & 3.9 & 1.68096 & 4. & 1.68586 & 4.1 & 1.6906 & 4.2 & 1.69518 \\
4.3 & 1.69963 & 4.4 & 1.70392 & 4.5 & 1.70809 & 4.6 & 1.71212 & 4.7 & 1.71603 & 4.8 & 1.71983 \\
4.9 & 1.72351 & 5. & 1.72709 & 5.1 & 1.73057 & 5.2 & 1.73395 & 5.3 & 1.73723 & 5.4 & 1.74043 \\
5.5 & 1.74354 & 5.6 & 1.74657 & 5.7 & 1.74952 & 5.8 & 1.75239 & 5.9 & 1.7552 & 6. & 1.75793 \\
6.1 & 1.76059 & 6.2 & 1.76319 & 6.3 & 1.76572 & 6.4 & 1.7682 & 6.5 & 1.77062 & 6.6 & 1.77298 \\
6.7 & 1.77529 & 6.8 & 1.77755 & 6.9 & 1.77975 & 7. & 1.78191 & 7.1 & 1.78402 & 7.2 & 1.78608 \\
\hline
\end{tabular}

\section{Numerical algorithm}

We compute the Konishi state energy in several steps. At the first step one solves the TBA equations for a fixed Bethe root $w$ by iterations. Equations for $Y_{ \pm}-$functions are solved first, then equations for $Y_{w}$ and $Y_{v w}$, and finally equations for $Y_{Q}$. For $g=0.7$ at the first iteration one uses the vacuum solution for $Y_{w^{-}}$and $Y_{v w}$-functions [31], and asymptotic $Y_{Q}$-functions [38]. ${ }^{10}$ For larger $g$ the solution found at the previous value of $g$, and the linear extrapolation of the Bethe root are used.

The number of iterations is bounded not to exceed $\bar{N}_{\text {iter }}=10$, and the iterations also stop if the absolute value of the difference between the energies of two successive iterations, $d E_{n}=\left|E_{n}-E_{n-1}\right|$, becomes less than $d e / 10$ where $d e=0.01$ is the precision the Konishi state energy is computed with at this step.

The number of $Y_{Q}$-functions computed is determined by the contribution of the asymptotic $Y_{Q}$-functions to the energy. It is given by $\int d \tilde{p} \log \left(1+Y_{Q}\right)$, and we solve the TBA equations only for those $Y_{Q}$-functions whose contributions exceed $10^{-5}$. We denote the total number of $Y_{Q^{-}}$functions used in the computation by $Q_{\max }$, and it depends on the value of $g$, to be precise we used, $Q_{\max }=3$ for $g=0.7, Q_{\max }=4$ for $0.8 \leq g \leq 1.1, Q_{\max }=5$ for $1.2 \leq g \leq 1.8, Q_{\max }=6$ for $1.9 \leq g \leq 2.8, Q_{\max }=7$ for $2.9 \leq g \leq 4.2$, and $Q_{\max }=8$ for $4.3 \leq g \leq 7.2$.

The number of $Y_{w}$-functions is equal to $N_{\mathrm{w}}=15$, and number of $Y_{v w}$-functions is equal to $N_{\mathrm{w}}+Q_{\max }-2$. This seems to be more than we need due to the locality of

\footnotetext{
${ }^{10}$ For $g<0.7$ we just use the asymptotic solution for $Y_{Q}$-functions. It is sufficient for the precision we are after. For small enough values of $g$ one can also use the asymptotic large $J$ solutions for Y-functions to start iterations. They are expressed in terms of transfer matrices corresponding to various representations of the symmetry algebra of the model under consideration [68, 69] (see also [30]). In the $\mathrm{AdS}_{5} \times \mathrm{S}^{5}$ case the symmetry algebra of the light-cone string theory is the centrally extended $\mathfrak{s u}(2 \mid 2)$ superalgebra [70,71], and explicit formulae for the transfer matrices were conjectured in [72] and derived in [73].
} 
the simplified TBA equations for auxiliary functions, and because the hybrid TBA equations for $Y_{Q^{-}}$function involve only $Y_{1 \mid v w^{-}}$and $Y_{Q-1 \mid v w^{-}}$functions.

Y-functions are computed at discrete sets of points. In particular, $Y_{Q}(u)$-functions are computed at points $u_{k}=k d u, k=0,1, \ldots$ with the step $d u=10 / 1001$ in the interval $\left[0, u_{\text {max }}^{q}(Q)\right]$, where $u_{\text {max }}^{q}(Q)$ is the lowest value of $u$ such that $Y_{Q}^{o}\left(u_{\text {max }}^{q}(Q)\right)<$ $10^{-6}$. Then, one uses Mathematica function Interpolation, and also the large $u$ behavior of asymptotic $Y_{Q}$-functions to define $Y_{Q}$ as continuous functions on the real $u$-line. $Y_{w^{-}}$(and $Y_{v w^{-}}$functions) are computed until $\left|1-Y_{Q \mid w}\left(u_{\max }^{w}(Q)\right) /(Q(Q+2))\right|<10^{-3}$ with a variable step equal to $d u$ for $0 \leq u<4$, to $2 d u$ for $4 \leq u<12$ and so on. One uses again Interpolation and the large $u$ asymptotic of $Y_{w}$ - and $Y_{v w}$-functions to define them for all $u$. Finally, $Y_{ \pm}(u)$-functions are computed at points $u_{k}=k d u_{y}$ with the step $d u_{y}=1 / 10$.

At the second step one uses the Y-functions to solve the exact Bethe equation, and find an adjusted value of $w$ and energy. Then one repeats the procedure until the difference between the energies becomes less than $d e=0.01$.

At the third step one uses the solution obtained to refine it by decreasing the steps $d u$ and $d u_{y}$, and the energy precision $d e$. We first solved the TBA equations with $d u \approx 1 / 50, d u_{y}=1 / 50$ and $d e=0.0001$. Then we decreased the steps to $d u \approx 1 / 100, d u_{y}=1 / 100$. Finally we used the steps $d u \approx 1 / 130, d u_{y}=1 / 130$ for $3.9 \leq g \leq 4.9$, and $d u \approx 1 / 150, d u_{y}=1 / 150$ for $g \geq 5.0$. The precision is about 0.0005 for $g<5.0$. For larger values of $g$ the precision decreases and is about 0.001 .

In version 2 of the paper to increase the precision we switched from the $u$ variable to the mirror momentum $\tilde{p}_{Q}$ rescaled so that the intervals $[0,2]$ are mapped to each other. We then used $g$-dependent steps $d \tilde{p}=2 / N_{0} \sqrt{2 / g}$ with $N_{0}=200$ and $N_{0}=$ 250. Finally, for $g>3$ we increased the number of auxiliary functions $N_{\mathrm{w}}=20$. Observing the changes in the energy, we believe that the final precision is about 0.0001 for all values of $g$.

To solve the hybrid TBA equations ${ }^{11}$ we compute the dressing kernels $K_{Q 1}^{\Sigma}(u, v)$ at the points $(k d u, n d u), k, n=0,1, \ldots$ with the step $d u=50 / 1001$, and $K_{Q Q^{\prime}}^{\Sigma}(u, v)$, $Q^{\prime} \geq 2$ with the step $d u=100 / 1001$ in the rectangle $\left[0, u_{\max }^{q}(Q)\right] \times\left[0, u_{\max }^{q}\left(Q^{\prime}\right)\right]$, and $K_{Q 1_{*}}^{\Sigma}(u, v)$ at the points $(k d u, \Delta v), k=0,1, \ldots$, where $\Delta v$ contains the Bethe root $w$, and the step in the $v$-direction is $d v=1 / 2000$.

\section{Dressing phases and kernels}

The improved dressing phases in various kinematic regions of the string and mirror models involve a common function which can be written as a sum of $\Phi$-functions, see [74] for definitions. We denote this function as $\Theta\left(x_{1}^{+}, x_{1}^{-}, u_{1}, Q, x_{2}^{+}, x_{2}^{-}, u_{2}, M, g\right)$,

\footnotetext{
${ }^{11}$ To avoid computing many dressing kernels we first tried to use the simplified TBA equations for $Y_{Q}$-functions. It appeared however that they were numerically unstable (at least with our algorithm) and led to a large systematic error increasing with $g$.
} 
where $x_{i}^{ \pm}$satisfy the usual constraints

$$
x_{1}^{ \pm}+\frac{1}{x_{1}^{ \pm}}=u_{1} \pm \frac{i}{g} Q, \quad x_{2}^{ \pm}+\frac{1}{x_{2}^{ \pm}}=u_{2} \pm \frac{i}{g} M .
$$

If the parameters $x_{i}^{ \pm}$belong to the string region, that is $\left|x_{i}^{ \pm}\right|>1$, then $\Theta$ is just equal to the BES dressing phase.

One can show by using the DHM representation [75] for the dressing phase that for any choice of $x_{i}^{ \pm}$satisfying (6.3), $\Theta$ has an integral representation involving elliptic $\Pi$-function. Since the derivation of the representation is rather involved we present below the expression leaving a proof of the formula to curious postdocs

$$
\begin{aligned}
& \Theta\left(x_{1}^{+}, x_{1}^{-}, u_{1}, Q, x_{2}^{+}, x_{2}^{-}, u_{2}, M, g\right)=\frac{i g^{2}}{\pi^{2}} \int_{0}^{1} \frac{d t}{2-t} \log \frac{\Gamma(1-2 i g(1-t))}{\Gamma(1+2 i g(1-t))}[ \\
& -\frac{i\left(\left(x_{1}^{-}\right)^{2}-1\right)\left(x_{2}^{-}-x_{2}^{+}\right)\left(x_{2}^{-} x_{2}^{+} Q+Q+i g\left(x_{2}^{-} x_{2}^{+} u_{1}+u_{1}-2\left(2 x_{2}^{-} x_{2}^{+}(1-t)+2(1-t)+x_{2}^{-}+x_{2}^{+}\right)\right)\right)}{\left(-i Q-2 g(1-t)+g u_{1}\right)\left(M^{2}-\left(Q+i g\left(-4(1-t)+u_{1}-u_{2}\right)\right)^{2}\right) x_{1}^{-} x_{2}^{-} x_{2}^{+}} \\
& \times \Pi\left(\frac{4 g^{2} t^{2}}{\left(-i Q-2 g(1-t)+g u_{1}\right)^{2}} \mid \frac{t^{2}}{(2-t)^{2}}\right) \\
& -\frac{i\left(\left(x_{1}^{+}\right)^{2}-1\right)\left(x_{2}^{-}-x_{2}^{+}\right)\left(x_{2}^{-} x_{2}^{+} Q+Q-i g\left(x_{2}^{-} x_{2}^{+} u_{1}+u_{1}-2\left(2 x_{2}^{-} x_{2}^{+}(1-t)+2(1-t)+x_{2}^{-}+x_{2}^{+}\right)\right)\right)}{\left(i Q-2 g(1-t)+g u_{1}\right)\left(M^{2}-\left(Q-i g\left(-4(1-t)+u_{1}-u_{2}\right)\right)^{2}\right) x_{2}^{-} x_{1}^{+} x_{2}^{+}} \\
& \times \Pi\left(\frac{4 g^{2} t^{2}}{\left(i Q-2 g(1-t)+g u_{1}\right)^{2}} \mid \frac{t^{2}}{(2-t)^{2}}\right) \\
& -\frac{\left(\left(x_{1}^{+}\right)^{2}-1\right)\left(x_{2}^{-}-x_{2}^{+}\right)\left(i Q\left(x_{2}^{-} x_{2}^{+}+1\right)+g\left(4(1-t)+u_{1}-2 x_{2}^{-}+\left(4(1-t) x_{2}^{-}+u_{1} x_{2}^{-}-2\right) x_{2}^{+}\right)\right)}{\left(-i Q-g\left(2(1-t)+u_{1}\right)\right)\left(M^{2}-\left(Q-i g\left(4(1-t)+u_{1}-u_{2}\right)\right)^{2}\right) x_{2}^{-} x_{1}^{+} x_{2}^{+}} \\
& \times \Pi\left(\frac{4 g^{2} t^{2}}{\left(-i Q-g\left(2(1-t)+u_{1}\right)\right)^{2}} \mid \frac{t^{2}}{(2-t)^{2}}\right) \\
& +\frac{\left(\left(x_{1}^{-}\right)^{2}-1\right)\left(x_{2}^{-}-x_{2}^{+}\right)\left(g\left(-4 t+u_{1}-2 x_{2}^{-}+\left(4(1-t) x_{2}^{-}+u_{1} x_{2}^{-}-2\right) x_{2}^{+}+4\right)-i Q\left(x_{2}^{-} x_{2}^{+}+1\right)\right)}{\left(i Q-g\left(2(1-t)+u_{1}\right)\right)\left(M^{2}-\left(Q+i g\left(4(1-t)+u_{1}-u_{2}\right)\right)^{2}\right) x_{1}^{-} x_{2}^{-} x_{2}^{+}} \\
& \times \Pi\left(\frac{4 g^{2} t^{2}}{\left(i Q-g\left(2(1-t)+u_{1}\right)\right)^{2}} \mid \frac{t^{2}}{(2-t)^{2}}\right) \\
& -\frac{\left(\left(x_{2}^{-}\right)^{2}-1\right)\left(x_{1}^{-}-x_{1}^{+}\right)\left(g\left(x_{1}^{-} x_{1}^{+} u_{2}+u_{2}-2\left(2 x_{1}^{-} x_{1}^{+}(1-t)+2(1-t)+x_{1}^{-}+x_{1}^{+}\right)\right)-i M\left(x_{1}^{-} x_{1}^{+}+1\right)\right)}{\left(Q^{2}-\left(M-i g\left(4(1-t)+u_{1}-u_{2}\right)\right)^{2}\right)\left(-i M-2 g(1-t)+g u_{2}\right) x_{1}^{-} x_{2}^{-} x_{1}^{+}} \\
& \times \Pi\left(\frac{4 g^{2} t^{2}}{\left(-i M-2 g(1-t)+g u_{2}\right)^{2}} \mid \frac{t^{2}}{(2-t)^{2}}\right) \\
& +\frac{\left(x_{1}^{-}-x_{1}^{+}\right)\left(i M\left(x_{1}^{-} x_{1}^{+}+1\right)+g\left(x_{1}^{-} x_{1}^{+} u_{2}+u_{2}-2\left(2 x_{1}^{-} x_{1}^{+}(1-t)+2(1-t)+x_{1}^{-}+x_{1}^{+}\right)\right)\right)\left(\left(x_{2}^{+}\right)^{2}-1\right)}{\left(Q^{2}-\left(M+i g\left(4(1-t)+u_{1}-u_{2}\right)\right)^{2}\right)\left(i M-2 g(1-t)+g u_{2}\right) x_{1}^{-} x_{1}^{+} x_{2}^{+}} \\
& \times \Pi\left(\frac{4 g^{2} t^{2}}{\left(i M-2 g(1-t)+g u_{2}\right)^{2}} \mid \frac{t^{2}}{(2-t)^{2}}\right) \\
& +\frac{\left(\left(x_{2}^{-}\right)^{2}-1\right)\left(x_{1}^{-}-x_{1}^{+}\right)\left(g\left(4(1-t)+u_{2}-2 x_{1}^{-}+\left(4(1-t) x_{1}^{-}+u_{2} x_{1}^{-}-2\right) x_{1}^{+}\right)-i M\left(x_{1}^{-} x_{1}^{+}+1\right)\right)}{\left(Q^{2}-\left(M-i g\left(-4(1-t)+u_{1}-u_{2}\right)\right)^{2}\right)\left(-i M+2 g(1-t)+g u_{2}\right) x_{1}^{-} x_{2}^{-} x_{1}^{+}} \\
& \times \Pi\left(\frac{4 g^{2} t^{2}}{\left(-i M+2 g(1-t)+g u_{2}\right)^{2}} \mid \frac{t^{2}}{(2-t)^{2}}\right) \\
& -\frac{\left(x_{1}^{-}-x_{1}^{+}\right)\left(i M\left(x_{1}^{-} x_{1}^{+}+1\right)+g\left(4(1-t)+u_{2}-2 x_{1}^{-}+\left(4(1-t) x_{1}^{-}+u_{2} x_{1}^{-}-2\right) x_{1}^{+}\right)\right)\left(\left(x_{2}^{+}\right)^{2}-1\right)}{\left(Q^{2}-\left(M+i g\left(-4(1-t)+u_{1}-u_{2}\right)\right)^{2}\right)\left(i M+2 g(1-t)+g u_{2}\right) x_{1}^{-} x_{1}^{+} x_{2}^{+}} \\
& \left.\times \Pi\left(\frac{4 g^{2} t^{2}}{\left(i M+2 g(1-t)+g u_{2}\right)^{2}} \mid \frac{t^{2}}{(2-t)^{2}}\right)\right] .
\end{aligned}
$$


This integral representation significantly speeds up computing the dressing phases. For small values of $g$ it might be useful to do the change $t \rightarrow(1-t) / g$ but for very large $g$ the representation seems to be the best one for numerics.

The function $\Theta$ is then used to define the corresponding expressions $\Theta_{s s}, \Theta_{s m}$, $\Theta_{m s}$ and $\Theta_{m m}$ in different regions of the $z$-torus

$$
\begin{aligned}
& \Theta_{s s}(u, Q, v, M, g)=\Theta\left(x_{s}\left(u+\frac{i Q}{g}\right), x_{s}\left(u-\frac{i Q}{g}\right), u, Q, x_{s}\left(v+\frac{i M}{g}\right), x_{s}\left(v-\frac{i M}{g}\right), v, M, g\right) \\
& \Theta_{s m}(u, Q, v, M, g)=\Theta\left(x_{s}\left(u+\frac{i Q}{g}\right), x_{s}\left(u-\frac{i Q}{g}\right), u, Q, x\left(v+\frac{i M}{g}\right), x\left(v-\frac{i M}{g}\right), v, M, g\right) \\
& \Theta_{m s}(u, Q, v, M, g)=\Theta\left(x\left(u+\frac{i Q}{g}\right), x\left(u-\frac{i Q}{g}\right), u, Q, x_{s}\left(v+\frac{i M}{g}\right), x_{s}\left(v-\frac{i M}{g}\right), v, M, g\right) \\
& \Theta_{m m}(u, Q, v, M, g)=\Theta\left(x\left(u+\frac{i Q}{g}\right), x\left(u-\frac{i Q}{g}\right), u, Q, x\left(v+\frac{i M}{g}\right), x\left(v-\frac{i M}{g}\right), v, M, g\right)
\end{aligned}
$$

Here $x(u)$ and $x_{s}(u)$ are the mirror and string $x$-functions: $x(u)=\frac{1}{2}\left(u-i \sqrt{4-u^{2}}\right)$, and $x_{s}(u)=\frac{1}{2} u\left(1+\sqrt{1-4 / u^{2}}\right)$. In particular, $\Theta_{s s}$ is just the BES dressing phase.

The improved dressing phases also involve contributions of $\Psi$-functions [74]. The total contribution of $\Psi\left(x_{2}, x_{1}\right)$-functions to the improved dressing phase in the stringmirror region is given by

$$
\begin{aligned}
& \Psi_{21}\left(x_{1}^{+}, x_{1}^{-}, u_{2}, g, M\right)=\int_{0}^{\pi / 2} d \theta \sin (\theta)[ \\
& \frac{i g M}{\pi\left(4\left(\frac{g u_{2}}{2}-g \cos (\theta)\right)^{2}+M^{2}\right)} \log \left(\frac{-i\left(x_{1}^{-}-x_{1}^{+}\right) \sin (\theta)-\left(x_{1}^{-}+x_{1}^{+}\right) \cos (\theta)+x_{1}^{-} x_{1}^{+}+1}{i\left(x_{1}^{-}-x_{1}^{+}\right) \sin (\theta)-\left(x_{1}^{-}+x_{1}^{+}\right) \cos (\theta)+x_{1}^{-} x_{1}^{+}+1}\right) \\
& -\frac{2 M}{4\left(\frac{g u_{2}}{2}+g \cos (\theta)\right)^{2}+M^{2}} \log \left(\frac{-i\left(x_{1}^{-}-x_{1}^{+}\right) \sin (\theta)+\left(x_{1}^{-}+x_{1}^{+}\right) \cos (\theta)+x_{1}^{-} x_{1}^{+}+1}{i\left(x_{1}^{-}-x_{1}^{+}\right) \sin (\theta)+\left(x_{1}^{-}+x_{1}^{+}\right) \cos (\theta)+x_{1}^{-} x_{1}^{+}+1}\right) \\
& +\left(\psi^{(0)}\left(\frac{M}{2}+\frac{i g u_{2}}{2}-i g \cos (\theta)+1\right)+\psi^{(0)}\left(\frac{M}{2}-\frac{i g u_{2}}{2}+i g \cos (\theta)+1\right)\right) \\
& \quad \times \log \left(\frac{-i\left(x_{1}^{-}-x_{1}^{+}\right) \sin (\theta)-\left(x_{1}^{-}+x_{1}^{+}\right) \cos (\theta)+x_{1}^{-} x_{1}^{+}+1}{i\left(x_{1}^{-}-x_{1}^{+}\right) \sin (\theta)-\left(x_{1}^{-}+x_{1}^{+}\right) \cos (\theta)+x_{1}^{-} x_{1}^{+}+1}\right) \\
& +\left(\psi^{(0)}\left(\frac{M}{2}-\frac{i g u_{2}}{2}-i g \cos (\theta)+1\right)+\psi^{(0)}\left(\frac{M}{2}+\frac{i g u_{2}}{2}+i g \cos (\theta)+1\right)\right) \\
& \left.\quad \times \log \left(\frac{-i\left(x_{1}^{-}-x_{1}^{+}\right) \sin (\theta)+\left(x_{1}^{-}+x_{1}^{+}\right) \cos (\theta)+x_{1}^{-} x_{1}^{+}+1}{i\left(x_{1}^{-}-x_{1}^{+}\right) \sin (\theta)+\left(x_{1}^{-}+x_{1}^{+}\right) \cos (\theta)+x_{1}^{-} x_{1}^{+}+1}\right)\right]
\end{aligned}
$$

Here the definitions are the same as above but $Q=1$, and $x_{1}^{ \pm}$are in the string region, that is $x_{1}^{ \pm}=x_{s}\left(u_{1} \pm \frac{i}{g}\right)$, while $x_{2}^{ \pm}$are in the mirror region, that is $x_{2}^{ \pm}=$ $x\left(u_{2} \pm \frac{i}{g} M\right)$. It is convenient to introduce

$$
\Psi_{21}^{\mathrm{sm}}\left(u_{1}, Q, u_{2}, M, g\right)=\Psi_{21}\left(x_{s}\left(u_{1}+\frac{i Q}{g}\right), x_{s}\left(u_{1}-\frac{i Q}{g}\right), u_{2}, g, M\right)
$$

Then, the contribution of the last term in the formula for the improved string-mirror 
dressing phase is given by

$$
\begin{aligned}
& \frac{1}{i} \log \Sigma_{\operatorname{last}}^{\mathrm{sm}}\left(u_{1}, Q, u_{2}, M, g\right)= \\
& \frac{1}{2 i} \log \frac{\left(x_{s}\left(u_{1}-\frac{i Q}{g}\right)-\frac{1}{x\left(u_{2}-\frac{i M}{g}\right)}\right)\left(x_{s}\left(u_{1}-\frac{i Q}{g}\right)-x\left(u_{2}+\frac{i M}{g}\right)\right)\left(x_{s}\left(u_{1}+\frac{i Q}{g}\right)-\frac{1}{x\left(u_{2}-\frac{i M}{g}\right)}\right)}{\left(x_{s}\left(u_{1}-\frac{i Q}{g}\right)-\frac{1}{x\left(u_{2}+\frac{i M}{g}\right)}\right)^{2}\left(x_{s}\left(u_{1}+\frac{i Q}{g}\right)-x\left(u_{2}+\frac{i M}{g}\right)\right)}
\end{aligned}
$$

The total improved dressing phase $\frac{1}{i} \log \Sigma_{1_{*} M}$ in the string-mirror region is thus given by the sum of the three terms

$$
\begin{aligned}
\frac{1}{i} \log \Sigma_{1_{*} M}\left(u_{1}, 1, u_{2}, M, g\right) & =\Theta^{\mathrm{sm}}\left(u_{1}, 1, u_{2}, M, g\right)+\Psi_{21}^{\mathrm{sm}}\left(u_{1}, 1, u_{2}, M, g\right) \\
& +\frac{1}{i} \log \Sigma_{\text {last }}^{\mathrm{sm}}\left(u_{1}, 1, u_{2}, M, g\right)
\end{aligned}
$$

The improved dressing phase in the mirror-string region is related to the one in the string-mirror region by the unitarity relation

$$
\frac{1}{i} \log \Sigma_{M 1_{*}}\left(u_{2}, u_{1}\right)=-\frac{1}{i} \log \Sigma_{1_{*} M}\left(u_{1}, u_{2}\right) .
$$

Finally, the improved dressing phase in the mirror-mirror region is given by the sum of four terms. The first one is $\Theta_{m m}$. The second term is the total contribution of $\Psi\left(x_{2}, x_{1}\right)$-functions given by

$$
\begin{aligned}
& \Psi_{21}^{\mathrm{m}}\left(x_{1}^{+}, x_{1}^{-}, u_{2}, g, M\right)=\frac{i}{2} \log \frac{\Gamma\left(-i g+\frac{1}{2} i\left(g u_{2}-i M\right)+1\right) \Gamma\left(-i g+\frac{1}{2} i\left(i M+g u_{2}\right)+1\right)}{\Gamma\left(i g-\frac{1}{2} i\left(g u_{2}-i M\right)+1\right) \Gamma\left(i g-\frac{1}{2} i\left(i M+g u_{2}\right)+1\right)} \\
& \quad+\frac{g}{2 \pi} \int_{0}^{\pi / 2} d \theta \sin (\theta)\left(-i \log \left(\frac{1-\frac{e^{-i \theta}}{x_{1}^{-}}}{1-\frac{e^{i \theta}}{x_{1}^{-}}}\right)-i \log \left(\frac{1-x_{1}^{+} e^{-i \theta}}{1-x_{1}^{+} e^{i \theta}}\right)+2 \theta-2 \pi\right) \\
& \times\left(-\frac{2 M}{4\left(\frac{g u_{2}}{2}-g \cos (\theta)\right)^{2}+M^{2}}+\psi^{(0)}\left(\frac{M}{2}+\frac{i g u_{2}}{2}-i g \cos (\theta)+1\right)+\psi^{(0)}\left(\frac{M}{2}-\frac{i g u_{2}}{2}+i g \cos (\theta)+1\right)\right) \\
& \quad+\sin (\theta)\left(-i \log \left(\frac{1+\frac{e^{i \theta}}{x_{1}^{-}}}{1+\frac{e^{-i \theta}}{x_{1}^{-}}}\right)-i \log \left(\frac{1+x_{1}^{+} e^{i \theta}}{1+x_{1}^{+} e^{-i \theta}}\right)-2 \theta\right) \\
& \times\left(-\frac{M}{4\left(\frac{g u_{2}}{2}+g \cos (\theta)\right)^{2}+M^{2}}+\psi^{(0)}\left(\frac{M}{2}-\frac{i g u_{2}}{2}-i g \cos (\theta)+1\right)+\psi^{(0)}\left(\frac{M}{2}+\frac{i g u_{2}}{2}+i g \cos (\theta)+1\right)\right)
\end{aligned}
$$


The third term is the total contribution of $\Psi\left(x_{1}, x_{2}\right)$-functions given by

$$
\begin{aligned}
& \Psi_{12}^{\mathrm{m}}\left(u_{1}, x_{2}^{+}, x_{2}^{-}, g, M\right)=-\frac{i}{2} \log \frac{\Gamma\left(-i g+\frac{1}{2} i\left(g u_{1}-i M\right)+1\right) \Gamma\left(-i g+\frac{1}{2} i\left(i M+g u_{1}\right)+1\right)}{\Gamma\left(i g-\frac{1}{2} i\left(g u_{1}-i M\right)+1\right) \Gamma\left(i g-\frac{1}{2} i\left(i M+g u_{1}\right)+1\right)} \\
& \quad+\frac{g}{2 \pi} \int_{0}^{\pi / 2} d \theta \sin (\theta)\left(i \log \left(\frac{1-\frac{e^{-i \theta}}{x_{-}^{-}}}{1-\frac{e^{i \theta}}{x_{2}^{-}}}\right)+i \log \left(\frac{1-x_{2}^{+} e^{-i \theta}}{1-x_{2}^{+} e^{i \theta}}\right)-2 \theta+2 \pi\right) \\
& \times\left(-\frac{2 M}{4\left(\frac{g u_{1}}{2}-g \cos (\theta)\right)^{2}+M^{2}}+\psi^{(0)}\left(\frac{M}{2}+\frac{i g u_{1}}{2}-i g \cos (\theta)+1\right)+\psi^{(0)}\left(\frac{M}{2}-\frac{i g u_{1}}{2}+i g \cos (\theta)+1\right)\right) \\
& \quad+\sin (\theta)\left(i \log \left(\frac{1+\frac{e^{i \theta}}{x_{2}^{-}}}{1+\frac{e^{-i \theta}}{x_{2}^{-}}}\right)+i \log \left(\frac{1+x_{2}^{+} e^{i \theta}}{1+x_{2}^{+} e^{-i \theta}}\right)+2 \theta\right) \\
& \times\left(-\frac{2 M}{4\left(\frac{g u_{1}}{2}+g \cos (\theta)\right)^{2}+M^{2}}+\psi^{(0)}\left(\frac{M}{2}-\frac{i g u_{1}}{2}-i g \cos (\theta)+1\right)+\psi^{(0)}\left(\frac{M}{2}+\frac{i g u_{1}}{2}+i g \cos (\theta)+1\right)\right)
\end{aligned}
$$

We also introduce

$$
\begin{aligned}
& \Psi_{12}^{\mathrm{mm}}\left(u_{1}, Q, u_{2}, M, g\right)=\Psi_{12}^{\mathrm{m}}\left(u_{1}, x\left(u_{2}+\frac{i M}{g}\right), x\left(u_{2}-\frac{i M}{g}\right), g, Q\right) \\
& \Psi_{21}^{\mathrm{mm}}\left(u_{1}, Q, u_{2}, M, g\right)=\Psi_{21}^{\mathrm{m}}\left(x\left(u_{1}+\frac{i Q}{g}\right), x\left(u_{1}-\frac{i Q}{g}\right), u_{2}, g, M\right)
\end{aligned}
$$

Finally, the last term is given by

$$
\begin{aligned}
& \frac{1}{i} \log \Sigma_{\text {last }}^{\operatorname{mm}}\left(u_{1}, Q, u_{2}, M, g\right)= \\
& \frac{1}{i} \log \left(\frac{i^{Q} \Gamma\left(M-\frac{1}{2} i\left(i(Q-M)+g u_{1}-g u_{2}\right)\right)\left(1-\frac{1}{x\left(u_{2}-\frac{i M}{g}\right) x\left(u_{1}+\frac{i Q}{g}\right)}\right)}{i^{M} \Gamma\left(Q+\frac{1}{2} i\left(i(Q-M)+g u_{1}-g u_{2}\right)\right)\left(1-\frac{1}{x\left(u_{2}+\frac{i M}{g}\right) x\left(u_{1}-\frac{i Q}{g}\right)}\right)} \sqrt{\frac{x\left(u_{2}-\frac{i M}{g}\right) x\left(u_{1}+\frac{i Q}{g}\right)}{x\left(u_{2}+\frac{i M}{g}\right) x\left(u_{1}-\frac{i Q}{g}\right)}}\right)
\end{aligned}
$$

Thus, the improved dressing phase $\Sigma_{Q M}$ in the mirror-mirror region is given by

$$
\begin{aligned}
\frac{1}{i} \log \Sigma_{Q M}\left(u_{1}, Q, u_{2}, M, g\right) & =\Theta^{\mathrm{mm}}\left(u_{1}, Q, u_{2}, M, g\right)+\Psi_{12}^{\mathrm{mm}}\left(u_{1}, Q, u_{2}, M, g\right)+\Psi_{21}^{\mathrm{mm}}\left(u_{1}, Q, u_{2}, M, g\right) \\
& +\frac{1}{i} \log \Sigma_{\text {last }}^{\mathrm{mm}}\left(u_{1}, Q, u_{2}, M, g\right)
\end{aligned}
$$

The formulae above are valid for any real $u_{1}, u_{2}$, and should be used with caution for complex values of the parameters.

Finally, the dressing kernels are defined as

$$
K_{Q M}^{\Sigma}(u, v)=\frac{1}{2 \pi i} \frac{d}{d u} \log \Sigma_{Q M}(u, v), \quad K_{Q 1_{*}}^{\Sigma}(u, v)=\frac{1}{2 \pi i} \frac{d}{d u} \log \Sigma_{Q 1_{*}}(u, v) .
$$

\section{Mathematica form of the dressing phases}

To make sure there are no misprints in the formulae above we also present below the same expressions in Mathematica input form.

Eq. (6.4) takes the form 


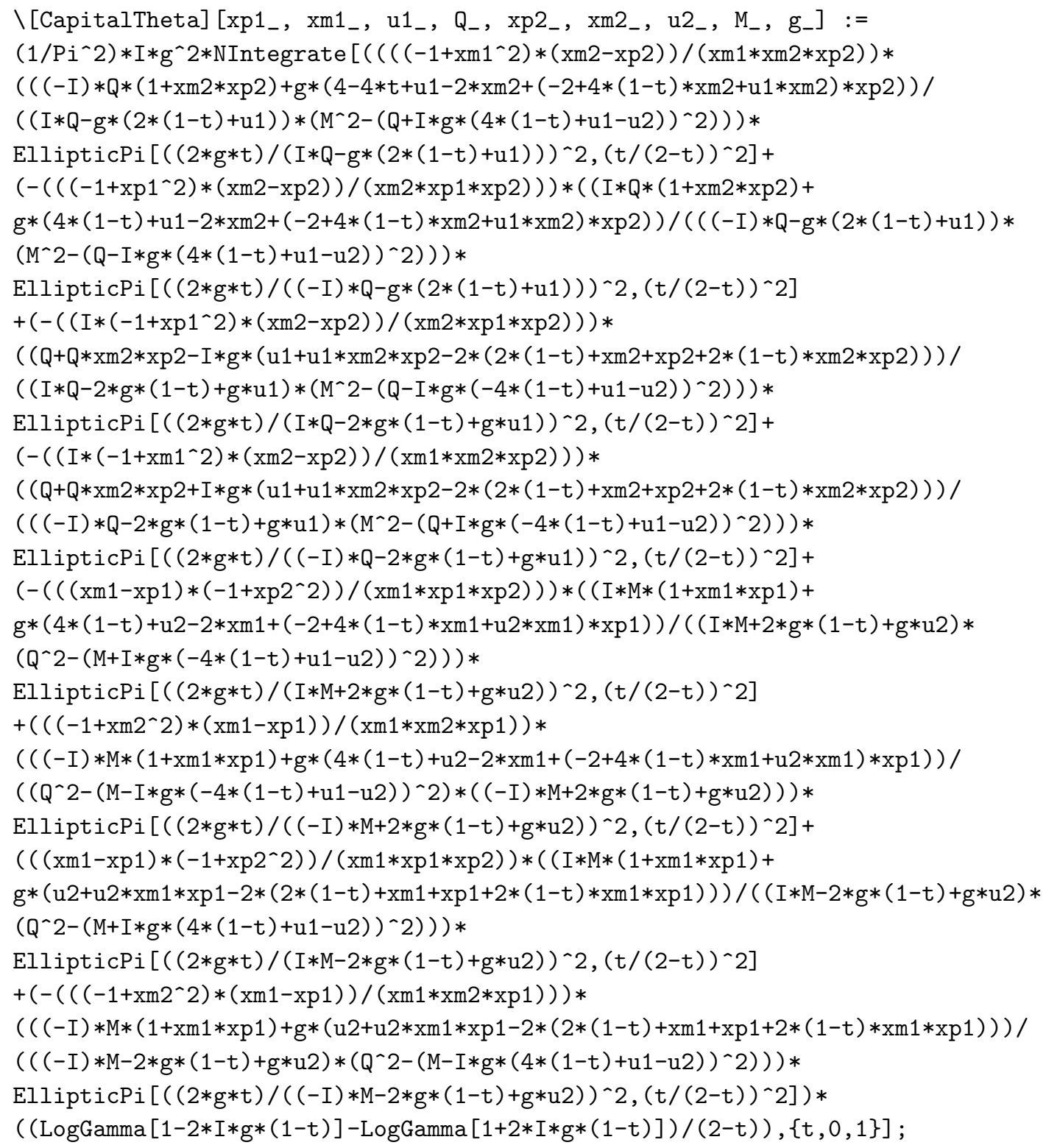

Here $\mathrm{xp} 1 \equiv x_{1}^{+}$, and so on, $x_{1}^{+}+1 / x_{1}^{+}=u_{1}+\frac{i}{g} Q$, and $x_{2}^{+}+1 / x_{2}^{+}=u_{2}+\frac{i}{g} M$. For effective numerics one should exclude the point $t=1-\frac{1}{4}\left|u_{1}-u_{2}\right|\left(\right.$ if $\left.\left|u_{1}-u_{2}\right|<4\right)$ from the integration region. This doubles the formula above, and by this reason we leave the necessary modification of the formula to the interested reader. For some points close to \pm 2 the formula works only if one specifies WorkingPrecision. For small values of $g$ and for the precision we are after it is sufficient to use WorkingPrecision $\rightarrow 10$. $\Theta_{s s}, \Theta_{s m}, \Theta_{m s}$ and $\Theta_{m m}$ in (6.5) are written as

$\backslash$ [CapitalTheta]ss $\left[u_{-}, Q_{-}, v_{-}, M_{-}, g_{-}\right]:=$

$\backslash$ [CapitalTheta] [xs [u+(I/g) Q], xs [u-(I/g) Q], u, Q, xs [v+(I/g)M], xs [v-(I/g)M],v, M, g] ;

$\backslash$ [CapitalTheta] sm $\left[u_{-}, Q_{-}, v_{-}, M_{-}, g_{-}\right]:=$

$\backslash$ [CapitalTheta] [xs [u+(I/g) Q], xs [u-(I/g) Q] , u, Q, x [v+(I/g)M], x [v-(I/g)M], v, M, g] ;

$\backslash$ [CapitalTheta]ms $\left[u_{-}, Q_{-}, V_{-}, M_{-}, g_{-}\right]:=$

$\backslash$ [CapitalTheta] $[\mathrm{x}[\mathrm{u}+(\mathrm{I} / \mathrm{g}) \mathrm{Q}], \mathrm{x}[\mathrm{u}-(\mathrm{I} / \mathrm{g}) \mathrm{Q}], \mathrm{u}, \mathrm{Q}, \mathrm{xs}[\mathrm{v}+(\mathrm{I} / \mathrm{g}) \mathrm{M}], \mathrm{xs}[\mathrm{v}-(\mathrm{I} / \mathrm{g}) \mathrm{M}], \mathrm{v}, \mathrm{M}, \mathrm{g}]$; 
$\backslash\left[\right.$ CapitalTheta] $\mathrm{mm}\left[\mathrm{u}_{-}, \mathrm{Q}_{-}, \mathrm{v}_{-}, \mathrm{M}_{-}, \mathrm{g}_{-}\right]:=$

$\backslash$ [CapitalTheta] $[\mathrm{x}[\mathrm{u}+(\mathrm{I} / \mathrm{g}) \mathrm{Q}], \mathrm{x}[\mathrm{u}-(\mathrm{I} / \mathrm{g}) \mathrm{Q}], \mathrm{u}, \mathrm{Q}, \mathrm{x}[\mathrm{v}+(\mathrm{I} / \mathrm{g}) \mathrm{M}], \mathrm{x}[\mathrm{v}-(\mathrm{I} / \mathrm{g}) \mathrm{M}], \mathrm{v}, \mathrm{M}, \mathrm{g}]$;

Here $x[u]$ and $x s[u]$ are the mirror and string $x$-functions.

The total contribution of $\Psi\left(x_{2}, x_{1}\right)$-functions to the improved dressing phase in the string-mirror region in (6.6) is given by

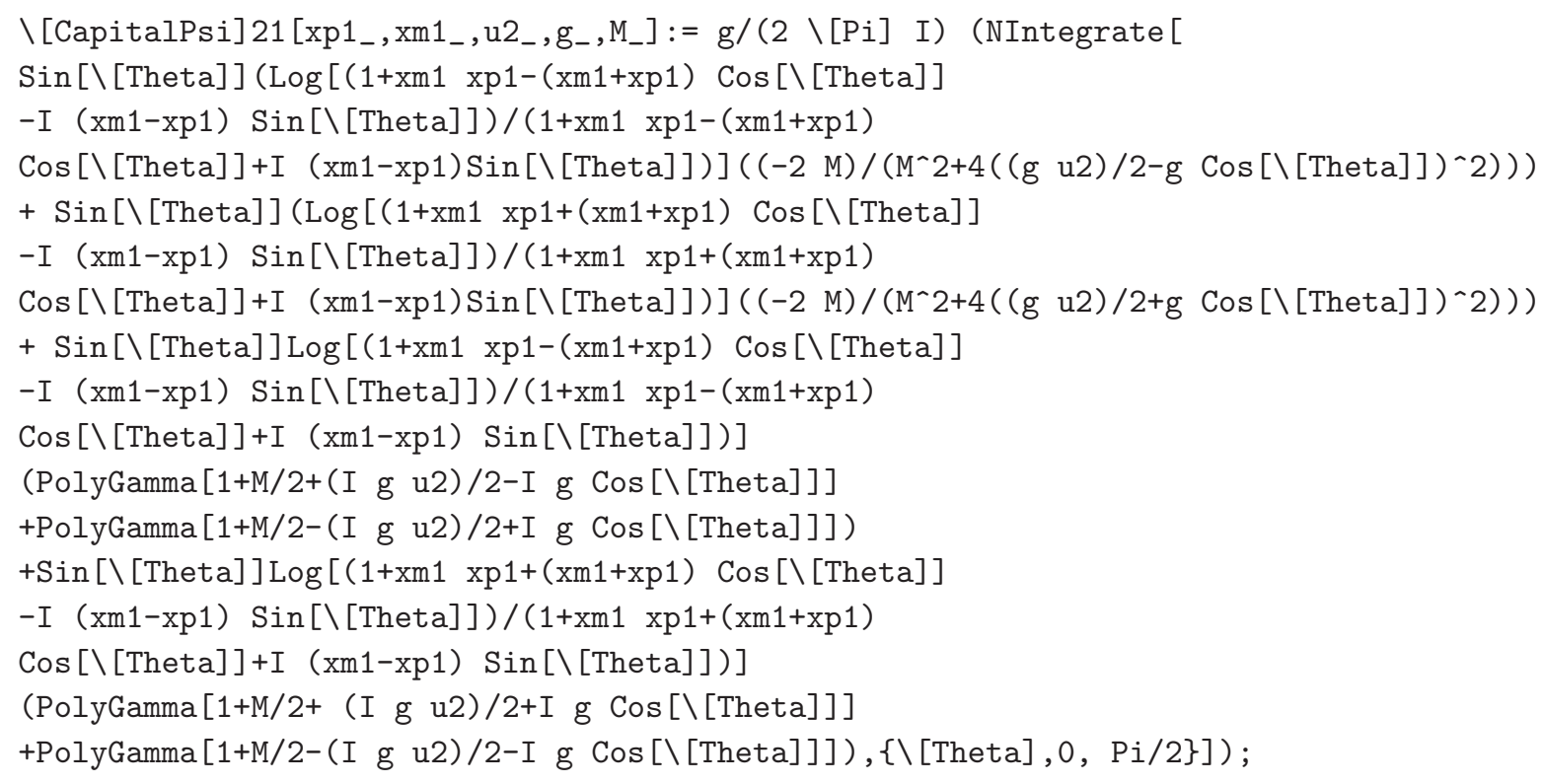

Here the definitions are the same as above but $Q=1$, and $x_{1}^{ \pm}$are in the string region, that is $x_{1}^{ \pm}=x_{s}\left(u_{1} \pm \frac{i}{g}\right)$, while $x_{2}^{ \pm}$are in the mirror region, that is $x_{2}^{ \pm}=$ $x\left(u_{2} \pm \frac{i}{g} M\right)$. Then, (6.7) is written as

$\backslash[$ CapitalSigma $] \backslash\left[\right.$ CapitalPsi]21[u1_, $\left.Q_{-}, u 2_{-}, M_{-}, g_{-}\right]:=$ $\backslash[$ CapitalPsi] $21[$ xs $[u 1+I *(Q / g)]$, xs $[u 1-I *(Q / g)], u 2, g, M]$;

The last term in the formula (6.8) for the improved string-mirror dressing phase is given by

$\backslash\left[\right.$ CapitalSigma] lastsm[u1 $\left., Q_{-}, u 2_{-}, M_{-}, g_{-}\right]=(1 /(2 * I)) * \log [(x s[u 1-I *(Q / g)]$

$-\mathrm{x}[\mathrm{u} 2+\mathrm{I} *(\mathrm{M} / \mathrm{g})]) *(\mathrm{xs}[\mathrm{u} 1-\mathrm{I} *(\mathrm{Q} / \mathrm{g})]-1 / \mathrm{x}[\mathrm{u} 2-\mathrm{I} *(\mathrm{M} / \mathrm{g})]) *((\mathrm{xs}[\mathrm{u} 1+\mathrm{I} *(\mathrm{Q} / \mathrm{g})]$

$-1 / \mathrm{x}[\mathrm{u} 2-\mathrm{I} *(\mathrm{M} / \mathrm{g})]) /((\mathrm{xs}[\mathrm{u} 1+\mathrm{I} *(\mathrm{Q} / \mathrm{g})]-\mathrm{x}[\mathrm{u} 2+\mathrm{I} *(\mathrm{M} / \mathrm{g})]) *(\mathrm{xs}[\mathrm{u} 1-\mathrm{I} *(\mathrm{Q} / \mathrm{g})]$

$-1 / x[u 2+I *(M / g)]) \wedge 2))]$;

The total improved dressing phase (6.9) in the string-mirror region is thus given by the sum of the three terms

$\backslash$ [CapitalSigma] Ism[u1 $\left., 1, \mathrm{u}_{-}, \mathrm{M}_{-}, \mathrm{g}_{-}\right]:=\backslash$ [CapitalTheta] $\mathrm{sm}[\mathrm{u} 1,1, \mathrm{u} 2, \mathrm{M}, \mathrm{g}]$ $+\backslash[$ CapitalSigma] \[CapitalPsi] 21[u1,1,u2,M,g] + \[CapitalSigma] lastsm[u1,1,u2, M,g];

The total contribution (6.11) of $\Psi\left(x_{2}, x_{1}\right)$-functions in the mirror-mirror region is 
$\backslash$ [CapitalPsi]21m[xp1 $\left., x m 1_{-}, u 2_{-}, g_{-}, M_{-}\right]:=$ $(\mathrm{g} /(2 * \mathrm{Pi})) * \mathrm{~N}$ Integrate $[\operatorname{Sin}[\backslash[$ Theta $]] *(2 * \backslash[$ Theta $]-2 * \mathrm{Pi}$

- $I * \log \left[\left(1-1 /\left(E^{\wedge}(I * \backslash[\right.\right.\right.$ Theta $\left.\left.]) * x m 1\right)\right) /\left(1-E^{\wedge}(I * \backslash[\right.$ Theta $\left.\left.]) / x m 1\right)\right]-$

$\left.\mathrm{I} * \log \left[\left(1-\operatorname{xp} 1 / \mathrm{E}^{\wedge}(\mathrm{I} * \backslash[\mathrm{Theta}])\right) /\left(1-\mathrm{E}^{\wedge}(\mathrm{I} * \backslash[\mathrm{Theta}]) * \mathrm{xp} 1\right)\right]\right) *\left((-2 * \mathrm{M}) /\left(\mathrm{M}^{\wedge} 2+4 *((\mathrm{~g} * \mathrm{u} 2) / 2\right.\right.$

- $\left.\mathrm{g} * \operatorname{Cos}[\backslash[\text { Theta }])^{\wedge} 2\right)+\operatorname{PolyGamma}[1+\mathrm{M} / 2+(I * \mathrm{~g} * \mathrm{u} 2) / 2-\mathrm{I} * \mathrm{~g} * \operatorname{Cos}[\backslash[$ Theta $]]]$

+ PolyGamma $[1+\mathrm{M} / 2-(\mathrm{I} * \mathrm{~g} * \mathrm{u} 2) / 2+\mathrm{I} * \mathrm{~g} * \operatorname{Cos}[\backslash[$ Theta $]]])+$

$\operatorname{Sin}[\backslash[$ Theta $]] *\left(-2 * \backslash[\right.$ Theta $]-I * \log \left[\left(1+E^{\wedge}(I * \backslash[\right.\right.$ Theta $\left.]) / x m 1\right) /\left(1+1 /\left(E^{\wedge}(I * \backslash[\right.\right.$ Theta $\left.\left.\left.]) * x m 1\right)\right)\right]-$ $\mathrm{I} * \log \left[\left(1+\mathrm{E}^{\wedge}(\mathrm{I} * \backslash[\right.\right.$ Theta $\left.]) * \operatorname{xp} 1\right) /\left(1+\operatorname{xp} 1 / \mathrm{E}^{\wedge}(\mathrm{I} * \backslash[\right.$ Theta $\left.\left.\left.])\right)\right]\right) *\left((-2 * \mathrm{M}) /\left(\mathrm{M}^{\wedge} 2+4 *((\mathrm{~g} * \mathrm{u} 2) / 2\right.\right.$ $\left.+\mathrm{g} * \operatorname{Cos}[\backslash[\text { Theta }])^{\wedge} 2\right)+$ PolyGamma $[1+\mathrm{M} / 2+(I * \mathrm{~g} * \mathrm{u} 2) / 2+\mathrm{I} * \mathrm{~g} * \operatorname{Cos}[\backslash[$ Theta $]]]+$ PolyGamma $[1+\mathrm{M} / 2-(\mathrm{I} * \mathrm{~g} * \mathrm{u} 2) / 2-\mathrm{I} * \mathrm{~g} * \operatorname{Cos}[\backslash[$ Theta $]]), \quad\{\backslash[$ Theta $], 0, \mathrm{Pi} / 2\}]+$ $(\mathrm{I} / 2) *(\operatorname{LogGamma}[1+(\mathrm{I} *(\mathrm{~g} * \mathrm{u} 2+\mathrm{I} * \mathrm{M})) / 2-\mathrm{I} * \mathrm{~g}]-\operatorname{LogGamma}[1-(\mathrm{I} *(\mathrm{~g} * \mathrm{u} 2+\mathrm{I} * \mathrm{M})) / 2+\mathrm{I} * \mathrm{~g}]+$ LogGamma $[1+(\mathrm{I} *(\mathrm{~g} * \mathrm{u} 2-\mathrm{I} * \mathrm{M})) / 2-\mathrm{I} * \mathrm{~g}]-\operatorname{LogGamma}[1-(\mathrm{I} *(\mathrm{~g} * \mathrm{u} 2-\mathrm{I} * \mathrm{M})) / 2+\mathrm{I} * \mathrm{~g}])$;

The total contribution (6.12) of $\Psi\left(x_{1}, x_{2}\right)$-functions is given by

$\backslash\left[\right.$ CapitalPsi]12m[u1 $\left., x p 2_{-}, x m 2_{-}, g_{-}, M_{-}\right]:=$

$(\mathrm{g} /(2 * \mathrm{Pi})) *$ NIntegrate $[\mathrm{Sin}[\backslash[$ Theta $]] *(-2 * \backslash[$ Theta $]+2 * \mathrm{Pi}$

$+\mathrm{I} * \log \left[\left(1-1 /\left(\mathrm{E}^{\wedge}(\mathrm{I} * \backslash[\right.\right.\right.$ Theta $\left.\left.]) * \mathrm{xm} 2\right)\right) /\left(1-\mathrm{E}^{\wedge}(\mathrm{I} * \backslash[\right.$ Theta $\left.\left.]) / \mathrm{xm} 2\right)\right]+$

$\left.\mathrm{I} * \log \left[\left(1-\operatorname{xp} 2 / \mathrm{E}^{\wedge}(\mathrm{I} * \backslash[\mathrm{Theta}])\right) /\left(1-\mathrm{E}^{\wedge}(\mathrm{I} * \backslash[\mathrm{Theta}]) * \operatorname{xp} 2\right)\right]\right) *\left((-2 * \mathrm{M}) /\left(\mathrm{M}^{\wedge} 2+4 *((\mathrm{~g} * \mathrm{u} 1) / 2\right.\right.$

$-\mathrm{g} * \operatorname{Cos}[\backslash[$ Theta $\left.]])^{\wedge} 2\right)+$ PolyGamma $[1+\mathrm{M} / 2+(\mathrm{I} * \mathrm{~g} * \mathrm{u} 1) / 2-\mathrm{I} * \mathrm{~g} * \operatorname{Cos}[\backslash[$ Theta $]]]$

+ PolyGamma $[1+\mathrm{M} / 2-(\mathrm{I} * \mathrm{~g} * \mathrm{u} 1) / 2+\mathrm{I} * \mathrm{~g} * \operatorname{Cos}[\backslash[$ Theta $]]])+\operatorname{Sin}[\backslash[$ Theta $]] *(2 * \backslash[$ Theta $]$

$+\mathrm{I} * \log \left[\left(1+\mathrm{E}^{\wedge}(\mathrm{I} * \backslash[\right.\right.$ Theta $\left.]) / \mathrm{xm} 2\right) /\left(1+1 /\left(\mathrm{E}^{\wedge}(\mathrm{I} * \backslash[\right.\right.$ Theta $\left.\left.\left.]) * \mathrm{xm} 2\right)\right)\right]$

$+\mathrm{I} * \log \left[\left(1+\mathrm{E}^{\wedge}(\mathrm{I} * \backslash[\right.\right.$ Theta $\left.]) * \operatorname{xp} 2\right) /\left(1+\mathrm{xp} 2 / \mathrm{E}^{\wedge}(\mathrm{I} * \backslash[\right.$ Theta $\left.\left.\left.])\right)\right]\right) *\left((-2 * \mathrm{M}) /\left(\mathrm{M}^{\wedge} 2+4 *((\mathrm{~g} * \mathrm{u} 1) / 2\right.\right.$

$+\mathrm{g} * \operatorname{Cos}[\backslash[$ Theta $\left.]])^{\wedge} 2\right)+$ PolyGamma $[1+\mathrm{M} / 2+(I * g * u 1) / 2+I * g * \operatorname{Cos}[\backslash[$ Theta $]]$

+ PolyGamma $[1+\mathrm{M} / 2-(\mathrm{I} * \mathrm{~g} * \mathrm{u} 1) / 2-\mathrm{I} * \mathrm{~g} * \operatorname{Cos}[\backslash[$ Theta $]]]),\{\backslash[$ Theta $], 0, \mathrm{Pi} / 2\}]-$

$(\mathrm{I} / 2) *(\operatorname{LogGamma}[1+(\mathrm{I} *(\mathrm{~g} * \mathrm{u} 1+\mathrm{I} * \mathrm{M})) / 2-\mathrm{I} * \mathrm{~g}]-\log G a m m a[1-(\mathrm{I} *(\mathrm{~g} * \mathrm{u} 1+\mathrm{I} * \mathrm{M})) / 2+\mathrm{I} * \mathrm{~g}]+$

LogGamma $[1+(\mathrm{I} *(\mathrm{~g} * \mathrm{u} 1-\mathrm{I} * \mathrm{M})) / 2-\mathrm{I} * \mathrm{~g}]-\operatorname{LogGamma}[1-(\mathrm{I} *(\mathrm{~g} * \mathrm{u} 1-\mathrm{I} * \mathrm{M})) / 2+\mathrm{I} * \mathrm{~g}])$;

$\Psi_{12}^{\mathrm{mm}}$ and $\Psi_{21}^{\mathrm{mm}}$ functions in (6.13) are

$\backslash\left[\right.$ CapitalSigma] $\backslash\left[\right.$ CapitalPsi] $12 \mathrm{~m}\left[\mathrm{u} 1_{-}, \mathrm{Q}_{-}, \mathrm{u} 2_{-}, \mathrm{M}_{-}, \mathrm{g}_{-}\right]$

$:=\backslash[$ CapitalPsi $] 12 \mathrm{~m}[\mathrm{u} 1, \mathrm{x}[\mathrm{u} 2+\mathrm{I} *(\mathrm{M} / \mathrm{g})], \mathrm{x}[\mathrm{u} 2-\mathrm{I} *(\mathrm{M} / \mathrm{g})], \mathrm{g}, \mathrm{Q}] ;$

$\backslash[$ CapitalSigma $] \backslash\left[\right.$ CapitalPsi] $21 \mathrm{~m}\left[\mathrm{u} 1_{-}, \mathrm{Q}_{-}, \mathrm{u} 2_{-}, \mathrm{M}_{-}, \mathrm{g}_{-}\right]$

$:=\backslash[$ CapitalPsi $] 21 \mathrm{~m}[\mathrm{x}[\mathrm{u} 1+\mathrm{I} *(\mathrm{Q} / \mathrm{g})], \mathrm{x}[\mathrm{u} 1-\mathrm{I} *(\mathrm{Q} / \mathrm{g})], \mathrm{u} 2, \mathrm{~g}, \mathrm{M}]$;

Finally, the last term (6.14) is given by

$\backslash$ [CapitalSigma] lastmm $\left[u 1_{-}, Q_{-}, u 2_{-}, M_{-}, g_{-}\right]:=$

(1/I) Log [( $\left.I^{\wedge} \mathrm{Q} / \mathrm{I}^{\wedge} \mathrm{M}\right)($ Gamma $[M-(\mathrm{I} / 2)(\mathrm{g} * \mathrm{u} 1-\mathrm{g} * \mathrm{u} 2+\mathrm{I}(\mathrm{Q}-\mathrm{M}))] /$ Gamma $[\mathrm{Q}+(\mathrm{I} / 2)(\mathrm{g} * \mathrm{u} 1-\mathrm{g} * \mathrm{u} 2+\mathrm{I}(\mathrm{Q}-\mathrm{M}))]) *$

$((1-1 /(\mathrm{x}[\mathrm{u} 1+\mathrm{I} *(\mathrm{Q} / \mathrm{g})] * \mathrm{x}[\mathrm{u} 2-\mathrm{I} *(\mathrm{M} / \mathrm{g})])) /(1-1 /(\mathrm{x}[\mathrm{u} 1-\mathrm{I} *(\mathrm{Q} / \mathrm{g})] * \mathrm{x}[\mathrm{u} 2+\mathrm{I} *(\mathrm{M} / \mathrm{g})]))) *$

$\left.((\mathrm{x}[\mathrm{u} 1+\mathrm{I} *(\mathrm{Q} / \mathrm{g})] / \mathrm{x}[\mathrm{u} 1-\mathrm{I} *(\mathrm{Q} / \mathrm{g})]) *(\mathrm{x}[\mathrm{u} 2-\mathrm{I} *(\mathrm{M} / \mathrm{g})] / \mathrm{x}[\mathrm{u} 2+\mathrm{I} *(\mathrm{M} / \mathrm{g})]))^{\wedge}(1 / 2)\right]$;

Thus, the improved dressing phase (6.15) in the mirror-mirror region is

$\backslash\left[\right.$ CapitalSigma] Imm $\left[u 1_{-}, Q_{-}, u 2_{-}, M_{-}, g_{-}\right]:=$

$\backslash$ [CapitalTheta]mm $[u 1, Q, u 2, M, g]+\backslash[$ CapitalSigma] $[$ CapitalPsi] $12 m[u 1, Q, u 2, M, g]$

$+\backslash[$ CapitalSigma] \[CapitalPsi]21m[u1,Q,u2, M, g] +\[CapitalSigma] lastmm [u1, Q, u2, M, g] ; 


\section{References}

[1] A. B. Zamolodchikov, "Thermodynamic Bethe Ansatz in Relativistic Models. Scaling Three State Potts and Lee-Yang Models," Nucl. Phys. B 342 (1990) 695.

[2] J. Ambjorn, R. A. Janik and C. Kristjansen, "Wrapping interactions and a new source of corrections to the spin-chain / string duality," Nucl. Phys. B 736 (2006) 288 [arXiv:hep-th/0510171].

[3] M. Luscher, "Volume Dependence of the Energy Spectrum in Massive Quantum Field Theories. 1. Stable Particle States," Commun. Math. Phys. 104 (1986) 177.

[4] G. Arutyunov and S. Frolov, "On String S-matrix, Bound States and TBA," JHEP 0712 (2007) 024, hep-th/0710.1568.

[5] N. Beisert and M. Staudacher, "Long-range $P S U(2,2 \mid 4)$ Bethe ansaetze for gauge theory and strings," Nucl. Phys. B 727 (2005) 1 [arXiv:hep-th/0504190].

[6] M. Takahashi, "One-Dimensional Hubbard Model at Finite Temperature," Prog. Theor. Phys. 47 (1972) 69.

[7] F.H.L. Essler, H. Frahm, F. Göhmann, A. Klümper, V.E. Korepin, "The One-Dimensional Hubbard Model," Cambridge University Press (2005).

[8] G. Arutyunov and S. Frolov, "String hypothesis for the $\mathrm{AdS}_{5} \times \mathrm{S}^{5}$ mirror," JHEP 0903 (2009) 152 [arXiv:0901.1417 [hep-th]].

[9] G. Arutyunov and S. Frolov, "Thermodynamic Bethe Ansatz for the $\mathrm{AdS}_{5} \times \mathrm{S}^{5}$ Mirror Model," JHEP 0905 (2009) 068 [arXiv:0903.0141 [hep-th]].

[10] D. Bombardelli, D. Fioravanti and R. Tateo, "Thermodynamic Bethe Ansatz for planar AdS/CFT: a proposal," J. Phys. A 42 (2009) 375401 [arXiv:0902.3930].

[11] N. Gromov, V. Kazakov, A. Kozak and P. Vieira, "Exact Spectrum of Anomalous Dimensions of Planar N $=4$ Supersymmetric Yang-Mills Theory: TBA and excited states," Lett. Math. Phys. 91 (2010) 265 [arXiv:0902.4458 [hep-th]].

[12] G. Arutyunov and S. Frolov, "Simplified TBA equations of the $\mathrm{AdS}_{5} \times \mathrm{S}^{5}$ mirror model," JHEP 0911 (2009) 019 [arXiv:0907.2647 [hep-th]].

[13] P. Dorey and R. Tateo, "Excited states by analytic continuation of TBA equations," Nucl. Phys. B 482 (1996) 639 [arXiv:hep-th/9607167].

[14] P. Dorey and R. Tateo, "Excited states in some simple perturbed conformal field theories," Nucl. Phys. B 515 (1998) 575 [arXiv:hep-th/9706140].

[15] V. V. Bazhanov, S. L. Lukyanov and A. B. Zamolodchikov, "Quantum field theories in finite volume: Excited state energies," Nucl. Phys. B 489 (1997) 487, hep-th/9607099. 
[16] P. Fendley, "Excited-state energies and supersymmetric indices," Adv. Theor. Math. Phys. 1 (1998) 210 [arXiv:hep-th/9706161].

[17] J. Balog and A. Hegedus, "TBA equations for excited states in the $O(3)$ and $O(4)$ nonlinear sigma-model," J. Phys. A 37 (2004) 1881 [arXiv:hep-th/0309009].

[18] A. Hegedus, "Nonlinear integral equations for finite volume excited state energies of the $O(3)$ and $O(4)$ nonlinear sigma-models," J. Phys. A 38 (2005) 5345 [arXiv:hep-th/0412125].

[19] A. Klümper, and P.A. Pearce, "Analytical calculations of Scaling Dimensions: Tricrit- ical Hard Square and Critical Hard Hexagons," J. Stat. Phys. 64, 13-76 (1991).

[20] A. Klümper, and P.A. Pearce, "Conformal weights of RSOS lattice models and their fusion hierarchies," J. Phys. A183, 304-350 (1992)

[21] C. Destri and H. J. de Vega, "New Thermodynamic Bethe Ansatz Equations Without Strings," Phys. Rev. Lett. 69 (1992) 2313.

[22] D. Fioravanti, A. Mariottini, E. Quattrini and F. Ravanini, "Excited state Destri-De Vega equation for sine-Gordon and restricted sine-Gordon models," Phys. Lett. B 390 (1997) 243 [arXiv:hep-th/9608091].

[23] C. Destri and H. J. de Vega, "Non-linear integral equation and excited-states scaling functions in the sine-Gordon model," Nucl. Phys. B 504 (1997) 621 [arXiv:hep-th/9701107].

[24] G. Feverati, F. Ravanini and G. Takacs, "Nonlinear integral equation and finite volume spectrum of sine-Gordon theory," Nucl. Phys. B 540 (1999) 543 [arXiv:hep-th/9805117].

[25] G. Feverati, F. Ravanini and G. Takacs, "Scaling functions in the odd charge sector of sine-Gordon/massive Thirring theory," Phys. Lett. B 444 (1998) 442 [arXiv:hep-th/9807160].

[26] J. Teschner, "On the spectrum of the Sinh-Gordon model in finite volume," Nucl. Phys. B 799 (2008) 403 [arXiv:hep-th/0702214].

[27] N. Gromov, V. Kazakov and P. Vieira, "Finite Volume Spectrum of 2D Field Theories from Hirota Dynamics," JHEP 0912 (2009) 060 [arXiv:0812.5091 [hep-th]].

[28] G. Arutyunov, S. Frolov and R. Suzuki, "Exploring the mirror TBA," arXiv:0911.2224 [hep-th].

[29] N. Gromov, V. Kazakov and P. Vieira, "Exact AdS/CFT spectrum: Konishi dimension at any coupling," arXiv:0906.4240 [hep-th]. 
[30] N. Gromov, V. Kazakov and P. Vieira, "Exact Spectrum of Anomalous Dimensions of Planar N=4 Supersymmetric Yang-Mills Theory," Phys. Rev. Lett. 103 (2009) 131601 [arXiv:0901.3753 [hep-th]].

[31] S. Frolov and R. Suzuki, "Temperature quantization from the TBA equations," Phys. Lett. B 679 (2009) 60 [arXiv:0906.0499 [hep-th]].

[32] A. Cavaglia, D. Fioravanti and R. Tateo, arXiv:1005.3016 [hep-th].

[33] G. Arutyunov, S. Frolov and R. Suzuki, "Five-loop Konishi from the Mirror TBA," arXiv:1002.1711 [hep-th].

[34] Z. Bajnok, A. Hegedus, R. A. Janik and T. Lukowski, "Five loop Konishi from AdS/CFT," arXiv:0906.4062 [hep-th].

[35] J. Balog and A. Hegedus, "5-loop Konishi from linearized TBA and the XXX magnet," arXiv:1002.4142 [hep-th].

[36] J. Balog and A. Hegedus, "The Bajnok-Janik formula and wrapping corrections," arXiv:1003.4303 [hep-th].

[37] T. Lukowski, A. Rej and V. N. Velizhanin, "Five-Loop Anomalous Dimension of Twist-Two Operators," arXiv:0912.1624 [hep-th].

[38] Z. Bajnok and R. A. Janik, "Four-loop perturbative Konishi from strings and finite size effects for multiparticle states," Nucl. Phys. B 807 (2009) 625 [arXiv:0807.0399 [hep-th]].

[39] F. Fiamberti, A. Santambrogio, C. Sieg and D. Zanon, "Wrapping at four loops in N=4 SYM," Phys. Lett. B 666 (2008) 100 [arXiv:0712.3522 [hep-th]].

[40] V. N. Velizhanin, "The Four-Loop Konishi in N=4 SYM," arXiv:0808.3832 [hep-th].

[41] J. M. Maldacena, "The large N limit of superconformal field theories and supergravity," Adv. Theor. Math. Phys. 2 (1998) 231 [Int. J. Theor. Phys. 38 (1999) 1113] [arXiv:hep-th/9711200].

[42] R. A. Janik and T. Lukowski, "Wrapping interactions at strong coupling - the giant magnon," Phys. Rev. D 76 (2007) 126008 [arXiv:0708.2208 [hep-th]].

[43] Y. Hatsuda and R. Suzuki, "Finite-Size Effects for Dyonic Giant Magnons," Nucl. Phys. B 800 (2008) 349 [arXiv:0801.0747 [hep-th]].

[44] J. A. Minahan and O. Ohlsson Sax, "Finite size effects for giant magnons on physical strings," Nucl. Phys. B 801 (2008) 97 [arXiv:0801.2064 [hep-th]].

[45] N. Gromov, S. Schafer-Nameki and P. Vieira, "Quantum Wrapped Giant Magnon," Phys. Rev. D 78 (2008) 026006 [arXiv:0801.3671 [hep-th]]. 
[46] Y. Hatsuda and R. Suzuki, "Finite-Size Effects for Multi-Magnon States," JHEP 0809 (2008) 025 [arXiv:0807.0643 [hep-th]].

[47] Z. Bajnok, R. A. Janik and T. Lukowski, "Four loop twist two, BFKL, wrapping and strings," Nucl. Phys. B 816 (2009) 376 [arXiv:0811.4448 [hep-th]].

[48] M. Beccaria, V. Forini, T. Lukowski and S. Zieme, "Twist-three at five loops, Bethe Ansatz and wrapping," JHEP 0903 (2009) 129 [arXiv:0901.4864 [hep-th]].

[49] M. Beccaria and G. F. De Angelis, "On the wrapping correction to single magnon energy in twisted N=4 SYM," arXiv:0903.0778 [hep-th].

[50] F. Fiamberti, A. Santambrogio and C. Sieg, "Five-loop anomalous dimension at critical wrapping order in N=4 SYM," arXiv:0908.0234 [hep-th].

[51] V. N. Velizhanin, "Six-Loop Anomalous Dimension of Twist-Three Operators in N=4 SYM," arXiv:1003.4717 [hep-th].

[52] D. H. Correa and C. A. S. Young, "Finite size corrections for open strings/open chains in planar AdS/CFT," JHEP 0908 (2009) 097 [arXiv:0905.1700 [hep-th]].

[53] A. Hegedus, "Discrete Hirota dynamics for AdS/CFT," Nucl. Phys. B 825 (2010) 341 [arXiv:0906.2546 [hep-th]].

[54] N. Gromov, "Y-system and Quasi-Classical Strings," JHEP 1001 (2010) 112 [arXiv:0910.3608 [hep-th]].

[55] D. Bombardelli, D. Fioravanti and R. Tateo, "TBA and Y-system for planar $A d S_{4} / C F T_{3}, "$ Nucl. Phys. B 834 (2010) 543 [arXiv:0912.4715 [hep-th]].

[56] N. Gromov and F. Levkovich-Maslyuk, "Y-system, TBA and Quasi-Classical Strings in AdS4 x CP3," arXiv:0912.4911 [hep-th].

[57] N. Gromov, V. Kazakov and Z. Tsuboi, "PSU $(2,2 \mid 4)$ Character of Quasiclassical AdS/CFT," arXiv:1002.3981 [hep-th].

[58] S. S. Gubser, I. R. Klebanov and A. M. Polyakov, "Gauge theory correlators from non-critical string theory," Phys. Lett. B 428 (1998) 105 [arXiv:hep-th/9802109].

[59] G. Arutyunov, S. Frolov and M. Staudacher, "Bethe ansatz for quantum strings," JHEP 0410 (2004) 016 [arXiv:hep-th/0406256].

[60] R. Roiban and A. A. Tseytlin, "Quantum strings in $\mathrm{AdS}_{5} \times \mathrm{S}^{5}$ : strong-coupling corrections to dimension of Konishi operator," JHEP 0911 (2009) 013 [arXiv:0906.4294 [hep-th]].

[61] G. Arutyunov and S. Frolov, "Uniform light-cone gauge for strings in $\mathrm{AdS}_{5} \times \mathrm{S}^{5}$ : Solving $\mathfrak{s u}(1 \mid 1)$ sector," JHEP 0601 (2006) 055 [arXiv:hep-th/0510208]. 
[62] N. Beisert, B. Eden and M. Staudacher, "Transcendentality and crossing," J. Stat. Mech. 0701 (2007) P021 [arXiv:hep-th/0610251].

[63] G. Arutyunov and S. Frolov, "Foundations of the $\mathrm{AdS}_{5} \times \mathrm{S}^{5}$ Superstring. Part I," J. Phys. A 42 (2009) 254003 [arXiv:0901.4937 [hep-th]].

[64] J. M. Maldacena and I. Swanson, "Connecting giant magnons to the pp-wave: An interpolating limit of $A d S_{5} \times S^{5}$," Phys. Rev. D 76 (2007) 026002 [arXiv:hep-th/0612079].

[65] M. Takahashi, M. Shiroishi, "TBA Equations of 1D Hubbard Model and High-Temperature Expansion," arXiv:cond-mat/0110645.

[66] M. Beccaria, "On the strong coupling expansion in the $s u(1 \mid 1)$ sector of $\mathcal{N}=4$ SYM," JHEP 0806 (2008) 063 [arXiv:0805.1180 [hep-th]].

[67] A. Rej and F. Spill, "Konishi at strong coupling from ABE," J. Phys. A 42 (2009) 442003 [arXiv:0907.1919 [hep-th]].

[68] A. Kuniba, T. Nakanishi and J. Suzuki, "Functional relations in solvable lattice models. 1: Functional relations and representation theory," Int. J. Mod. Phys. A 9 (1994) 5215 [arXiv:hep-th/9309137].

[69] Z. Tsuboi, "A note on the osp $(1 \mid 2 s)$ thermodynamic Bethe ansatz equation," Int. J. Mod. Phys. A 17, 2351 (2002) [arXiv:cond-mat/0108358].

[70] N. Beisert, "The su(2|2) dynamic S-matrix," Adv. Theor. Math. Phys. 12 (2008) 945 [arXiv:hep-th/0511082].

[71] G. Arutyunov, S. Frolov, J. Plefka and M. Zamaklar, "The off-shell symmetry algebra of the light-cone AdS(5) x S**5 superstring," J. Phys. A 40 (2007) 3583 [arXiv:hep-th/0609157].

[72] N. Beisert, "The Analytic Bethe Ansatz for a Chain with Centrally Extended su(2|2) Symmetry," J. Stat. Mech. 0701 (2007) P017 [arXiv:nlin/0610017].

[73] G. Arutyunov, M. de Leeuw, R. Suzuki and A. Torrielli, "Bound State Transfer Matrix for $\mathrm{AdS}_{5} \times \mathrm{S}^{5}$ Superstring," JHEP 0910, 025 (2009) [arXiv:0906.4783].

[74] G. Arutyunov and S. Frolov, "The Dressing Factor and Crossing Equations," J. Phys. A 42 (2009) 425401 [arXiv:0904.4575 [hep-th]].

[75] N. Dorey, D. M. Hofman and J. M. Maldacena, "On the singularities of the magnon S-matrix," Phys. Rev. D 76 (2007) 025011 [arXiv:hep-th/0703104]. 